\title{
On the homotopy groups of symmetric spectra
}

\author{
STEFAN SCHWEDE
}

\begin{abstract}
We construct a natural, tame action of the monoid of injective self-maps of the set of natural numbers on the homotopy groups of a symmetric spectrum. This extra algebraic structure allows a conceptual and uniform understanding of various phenomena related to $\pi_{*}$-isomorphisms, semistability and the relationship between naive and true homotopy groups for symmetric spectra.
\end{abstract}

$55 \mathrm{P} 42 ; 55 \mathrm{U} 35$

Symmetric spectra are an easy-to-define and convenient model for the stable homotopy category with a nice smash product. Symmetric ring spectra first showed up under the name "FSP on spheres" in the context of algebraic $K$-theory and topological Hochschild homology. Around 1993, Jeff Smith made the crucial observation that the "FSPs on spheres" are the monoids in a category of "symmetric spectra" with respect to an associative and commutative smash product, and he suspected compatible model category structures so that one obtains as homotopy categories "the" stable homotopy category (for symmetric spectra), the homotopy category of $A_{\infty}$ ring spectra (for symmetric ring spectra), respectively the homotopy category of $E_{\infty}$ ring spectra (for commutative symmetric ring spectra). The details of various model structures were worked out by Hovey, Shipley and Smith in [3].

Maybe the only tricky point with symmetric spectra is that the stable equivalences can not be defined by looking at stable homotopy groups (defined as the classical sequential colimit of the unstable homotopy groups of the terms in a symmetric spectrum). Formally inverting the $\pi_{*}$-isomorphisms, ie, those morphisms which induce isomorphisms of stable homotopy groups, leaves too many homotopy types. Instead, Hovey, Shipley and Smith introduce a strictly larger class of stable equivalences, defined as the morphisms which induce isomorphisms on all cohomology theories. The difference between $\pi_{*}$-isomorphisms and stable equivalences has previously confused at least the present author. The precise relationship between the naively defined homotopy groups and the "true" homotopy groups (morphisms from sphere in the stable homotopy category) has largely been mysterious (although Shipley's detection functor [7, Section 3] sheds considerable light on this).

In this paper we advertise and systematically exploit extra algebraic structure on the (classical) homotopy groups of a symmetric spectrum which, in the authors opinion, 
clarifies several otherwise ad hoc observations and illuminates various mysterious points in the theory of symmetric spectra. This extra structure is an action of the monoid $M$ of injective self-maps of the set of natural numbers. The $M$-modules that come up, however, have a special property which we call tameness; see Definition 1.4. Tameness has strong algebraic consequences and severely restricts the kinds of $M$-modules which can arise as homotopy groups of symmetric spectra.

Here is a first example of the use of the $M$-action. An important class of symmetric spectra is formed by the semistable symmetric spectra. Within this class, stable equivalences coincide with $\pi_{*}$-isomorphisms, so it is very useful to recognize a given symmetric spectrum as semistable. In Theorem 4.1, we characterize the semistable symmetric spectra as those for which the $M$-action on homotopy groups is trivial. Other examples for the use of the $M$-action are the following:

- The group $\pi_{0}\left(F_{n} S^{n}\right)$ is not just an infinitely generated free abelian group, but a very prominent tame $M$-module $\mathcal{P}_{n}$; these $M$-modules are pairwise nonisomorphic for different $n$ (Example 3.4).

- Certain monomorphisms on homotopy groups which appear in the discussion of semistable symmetric spectra are given by the action of the special element $d$ of $M$ defined by $d(i)=i+1$ (Example 1.6).

- A tame $M$-module which is finitely generated as an abelian group necessarily has trivial $M$-action (Lemma 2.3 (iv)); this implies that symmetric spectra whose homotopy groups are dimensionwise finitely generated are always semistable. This puts [3, Proposition 5.6.4 (1)] into perspective, which proves that dimensionwise finite homotopy groups implies semistability.

- The homotopy groups of the symmetric spectra $\Omega X$ and $S^{1} \wedge X$ (Example 3.10), sh $X$ (Example 3.11), $F_{1} S^{0} \wedge X$ (Example 3.13), $R X$ and $R^{\infty} X$ (Example $3.15)$ are functors of the homotopy groups of $X$, and the $M$-action on $\pi_{*} X$ determines the $M$-action on the homotopy groups of these constructions.

- The $E^{2}$-term of Shipley's Bousfield-Kan spectral sequence for calculating the homotopy groups of $D X$ can be identified with the Tor groups of $\pi_{*} X$ over the monoid ring of $M$, and the spectral sequence can be analyzed in many examples (Section 5).

The $M$-action is also intertwined in an interesting and nontrivial way with the smash product of symmetric spectra. This explains the curious phenomenon that the homotopy groups of a symmetric ring spectrum do in general not form a graded ring in any natural way (unless the underlying symmetric spectrum in semistable); the problem is that the termwise smash product pairings are not compatible with the stabilization maps in 
the colimit defining stable homotopy groups. The natural algebraic structure on the homotopy of a symmetric ring spectrum is an action by the injection operad of the set of natural numbers. This operad is a discrete analog, with similar properties, of the operad of linear isometries which features prominently in other foundational parts of stable homotopy theory. We do not discuss these topics here but hope to return to them elsewhere.

Acknowledgments Several of the things which we discuss are contained, mostly implicitly, in the papers by Hovey, Shipley and Smith [3] and Shipley [7]. The $M-$ action makes a brief appearance in Proposition 2.2.9 of [7], which Shipley attributes to Smith; it appears though that the $M$-action has not been used systematically, nor has tameness been exploited before.

\section{Homotopy groups as $M$-modules}

A symmetric spectrum consists of the following data:

- a sequence of pointed spaces $X_{n}$ for $n \geq 0$;

- a base-point preserving continuous left action of the symmetric group $\Sigma_{n}$ on $X_{n}$, for each $n \geq 0$;

- pointed continuous maps $\sigma_{n}: X_{n} \wedge S^{1} \longrightarrow X_{n+1}$ for $n \geq 0$.

This data is subject to the following condition: the composite

$$
X_{n} \wedge S^{m} \stackrel{\sigma_{n} \wedge \mathrm{Id}}{\longrightarrow} X_{n+1} \wedge S^{m-1} \stackrel{\sigma_{n+1} \wedge \mathrm{Id}}{\longrightarrow} \cdots \stackrel{\sigma_{n+m-1}}{\longrightarrow} X_{n+m}
$$

is $\Sigma_{n} \times \Sigma_{m}$-equivariant for all $n, m \geq 0$. Here $S^{m}$ is the $m$-fold smash product of $S^{1}$, on which the symmetric group $\Sigma_{m}$ acts by permuting the factors, and the action on the target $X_{n+m}$ is by restriction from $\Sigma_{n+m}$ to the subgroup $\Sigma_{n} \times \Sigma_{m}$; here and in the rest of the paper we view $\Sigma_{n} \times \Sigma_{m}$ as a subgroup of $\Sigma_{n+m}$ via the monomorphism which sends $(\tau, \delta) \in \Sigma_{n} \times \Sigma_{m}$ to $\tau \times \delta \in \Sigma_{n+m}$ defined by

$$
(\tau \times \delta)(i)=\left\{\begin{array}{cl}
\tau(i) & \text { for } 1 \leq i \leq n, \text { and } \\
\delta(i-n)+n & \text { for } n+1 \leq i \leq n+m .
\end{array}\right.
$$

A morphism $f: X \longrightarrow Y$ of symmetric spectra consists of $\Sigma_{n}$-equivariant continuous pointed maps $f_{n}: X_{n} \longrightarrow Y_{n}$ for $n \geq 0$, which are compatible with the structure maps in the sense that $f_{n+1} \circ \sigma_{n}=\sigma_{n} \circ\left(f_{n} \wedge \mathrm{Id}\right)$ for all $n \geq 0$. The category of symmetric spectra is denoted by $S p^{\Sigma}$. 
The $k$-th stable homotopy group of a symmetric spectrum $X$, for $k$ any integer, is the colimit

$$
\pi_{k} X=\operatorname{colim}_{n} \pi_{k+n} X_{n},
$$

taken over the maps $\iota_{*}: \pi_{k+n} X_{n} \longrightarrow \pi_{k+n+1} X_{n+1}$ defined as the composite

$$
\pi_{k+n} X_{n} \stackrel{-\wedge S^{1}}{\longrightarrow} \pi_{k+n+1}\left(X_{n} \wedge S^{1}\right) \stackrel{\left(\sigma_{n}\right)_{*}}{\longrightarrow} \pi_{k+n+1} X_{n+1} .
$$

A $\pi_{*}$-isomorphism is a morphism of symmetric spectra which induces an isomorphism on all stable homotopy groups. Every $\pi_{*}$-isomorphism is a stable equivalence in the sense of [3, Definition 3.1.3] respectively [5, Definition 8.3], but not conversely. Inverting only the $\pi_{*}$-isomorphisms of symmetric spectra leaves too many homotopy types, and the resulting category is not equivalent to the usual stable homotopy category.

The definition of homotopy groups does not take the symmetric group actions into account; using these actions we will now see that the homotopy groups of a symmetric spectrum have more structure.

1.2 Construction We define an action of the monoid $M$ of injective self-maps of the set $\omega=\{1,2,3, \ldots\}$ of positive natural numbers, on the homotopy groups of a symmetric spectrum. We break the construction up into two steps and pass through the intermediate category of $I$-functors. The category $I$ has an object $\mathbf{n}=\{1, \ldots, n\}$ for every nonnegative integer $n$, including $\mathbf{0}=\varnothing$. Morphisms in $I$ are all injective maps. An $I$-functor is a covariant functor from $I$ to the category of abelian groups.

Step 1 (from symmetric spectra to $I$-functors) For every integer $k$ we assign an $I$-functor $\underline{\pi}_{k} X$ to a symmetric spectrum $X$. On objects, this $I$-functor is given by

$$
\left(\underline{\pi}_{k} X\right)(\mathbf{n})=\pi_{k+n} X_{n}
$$

if $k+n \geq 2$ and $\left(\underline{\pi}_{k} X\right)(\mathbf{n})=0$ for $k+n<2$. If $\alpha: \mathbf{n} \longrightarrow \mathbf{m}$ is an injective map and $k+n \geq 2$, then $\alpha_{*}:\left(\underline{\pi}_{k} X\right)(\mathbf{n}) \longrightarrow\left(\underline{\pi}_{k} X\right)(\mathbf{m})$ is given as follows. We choose a permutation $\gamma \in \Sigma_{m}$ such that $\gamma(i)=\alpha(i)$ for all $i=1, \ldots, n$ and set

$$
\alpha_{*}(x)=\operatorname{sgn}(\gamma) \cdot \gamma_{*}\left(\iota_{*}^{m-n}(x)\right)
$$

where $\iota_{*}: \pi_{k+n} X_{n} \longrightarrow \pi_{k+n+1} X_{n+1}$ is the stabilization map (1.1).

We have to justify that this definition is independent of the choice of permutation $\gamma$. Suppose $\gamma^{\prime} \in \Sigma_{m}$ is another permutation which agrees with $\alpha$ on $\mathbf{n}$. Then $\gamma^{-1} \gamma^{\prime}$ is a permutation of $\mathbf{m}$ which fixes the numbers $1, \ldots, n$, so it is of the form $\gamma^{-1} \gamma^{\prime}=1 \times \tau$ for some $\tau \in \Sigma_{m-n}$, where 1 is the unit of $\Sigma_{n}$. It suffices to show that for such 
permutations the induced action on $\pi_{k+m} X_{m}$ via the action on $X_{m}$ satisfies the relation

$$
(1 \times \tau)_{*}\left(\iota_{*}^{m-n}(x)\right)=\operatorname{sgn}(\tau) \cdot\left(\iota_{*}^{m-n}(x)\right)
$$

for all $x \in \pi_{k+n} X_{n}$. To justify this we let $f: S^{k+n} \longrightarrow X_{n}$ represent $x$. Since the iterated structure map $\sigma^{m-n}: X_{n} \wedge S^{m-n} \longrightarrow X_{m}$ is $\Sigma_{n} \times \Sigma_{m-n}$-equivariant, we have a commutative diagram:

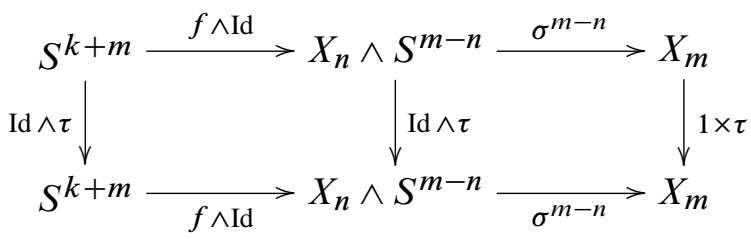

The composite through the upper right corner represents $(1 \times \tau)_{*}\left(\iota_{*}^{m-n}(x)\right)$. Since the effect on homotopy groups of precomposing with a coordinate permutation of the sphere is multiplication by the sign of the permutation, the composite through the lower left corner represents $\operatorname{sgn}(\tau) \cdot\left(\iota_{*}^{m-n}(x)\right)$. This proves formula (1.3) and completes the definition of $\alpha_{*}:\left(\underline{\pi}_{k} X\right)(\mathbf{n}) \longrightarrow\left(\underline{\pi}_{k} X\right)(\mathbf{m})$.

The inclusion $\mathbf{n} \longrightarrow \mathbf{n}+\mathbf{1}$ induces the map $\iota_{*}$ over which the colimit $\pi_{k} X$ is formed, so if we denote the inclusion by $\iota$, then the two meanings of $\iota_{*}$ are consistent. We let $\mathbb{N}$ denote the subcategory of $I$ which contains all objects but only the inclusions as morphisms, and then we have

$$
\pi_{k} X=\operatorname{colim}_{\mathbb{N}} \underline{\pi}_{k} X .
$$

Step 2 (from $I$-functors to tame $M$-modules) The next observation is that for any $I$-functor $F$ the colimit of $F$, formed over the subcategory $\mathbb{N}$ of inclusions, has a natural left action by the monoid $M$ of injective self-maps of the set $\omega$ of natural numbers. Applied to the $I$-functor $\underline{\pi}_{k} X$ coming from a symmetric spectrum $X$, this yields the $M$-action on the stable homotopy group $\pi_{k} X$.

We let $I_{\omega}$ denote the category with objects the sets $\mathbf{n}$ for $n \geq 0$ and the set $\omega$ and with all injective maps as morphisms. So $I_{\omega}$ contains $I$ as a full subcategory and contains one more object $\omega$ whose endomorphism monoid is $M$. We will now extend an $I$-functor $F$ to a functor from the category $I_{\omega}$ in such a way that the value of the extension at the object $\omega$ is the colimit of $F$, formed over the subcategory $\mathbb{N}$ of inclusions. It will thus be convenient, and suggestive, to denote the colimit of $F$, formed over the subcategory $\mathbb{N}$ of inclusions, by $F(\omega)$ and not introduce new notation for the extended functor. The $M$-action on the colimit of $F$ is then the action of the endomorphisms of $\omega$ in $I_{\omega}$ on $F(\omega)$. 
So we set $F(\omega)=\operatorname{colim}_{\mathbb{N}} F$ and first define $\beta_{*}: F(\mathbf{n}) \longrightarrow F(\omega)$ for every injection $\beta: \mathbf{n} \longrightarrow \omega$ as follows. We set $m=\max \{\beta(\mathbf{n})\}$, denote by $\left.\beta\right|_{\mathbf{n}}: \mathbf{n} \longrightarrow \mathbf{m}$ the restriction of $\beta$ and take $\beta_{*}(x)$ to be the class in the colimit represented by the image of $x$ under

$$
\left(\left.\beta\right|_{\mathbf{n}}\right)_{*}: F(\mathbf{n}) \longrightarrow F(\mathbf{m})
$$

It is straightforward to check that this is a functorial extension of $F$, ie, for every morphism $\alpha: \mathbf{k} \longrightarrow \mathbf{n}$ in $I$ we have $(\beta \alpha)_{*}(x)=\beta_{*}\left(\alpha_{*}(x)\right)$.

Now we let $f: \omega \longrightarrow \omega$ be an injective self-map of $\omega$, and we want to define $f_{*}: F(\omega) \longrightarrow F(\omega)$. If $[x] \in F(\omega)$ is an element in the colimit represented by a class $x \in F(\mathbf{n})$, then we set $f_{*}[x]=\left[\left(\left.f\right|_{\mathbf{n}}\right)_{*}(x)\right]$ where $\left.f\right|_{\mathbf{n}}: \mathbf{n} \longrightarrow \omega$ is the restriction of $f$ and $f_{*}: F(\mathbf{n}) \longrightarrow F(\omega)$ was defined in the previous paragraph. Again it is straightforward to check that this definition does not depend in the representative $x$ of the class $[x]$ in the colimit and that the extension is functorial, ie, we have $(f \alpha)_{*}(x)=f_{*}\left(\alpha_{*}(x)\right)$ for injections $\alpha: \mathbf{n} \longrightarrow \omega$ as well as $(f g)_{*}[x]=f_{*}\left(g_{*}[x]\right)$ when $g$ is another injective self-map of $\omega$. As an example, if we also write $\iota: \mathbf{n} \longrightarrow \omega$ for the inclusion, then we have $\iota_{*}(x)=[x]$ for $x \in F(\mathbf{n})$.

The definition just given is in fact the universal way to extend an $I$-functor $F$ to a functor on the category $I_{\omega}$, ie, we have just constructed a left Kan extension of $F: I \longrightarrow \mathcal{A} b$ along the inclusion $I \longrightarrow I_{\omega}$. However, we do not need this fact, so we omit the proof.

A trivial but important observation straight from the definition is that the action of the monoid $M$ on the colimit of any $I$-functor $F$ has a special property: every element in the colimit $F(\omega)$ is represented by a class $x \in F(\mathbf{n})$ for some $n \geq 0$; then for every element $f \in M$ which fixes the numbers $1, \ldots, n$, we have $f_{*}[x]=[x]$. We introduce a special name for such $M$-modules:

1.4 Definition An $M$-module is a left module over the monoid ring $\mathbb{Z}[M]$ of the monoid $M$ of injective self-maps of the set $\omega=\{1,2,3, \ldots\}$. We call an $M$-module $W$ tame if for every element $x \in W$ there exists a number $n \geq 0$ with the following property: for every element $f \in M$ which fixes the set $\mathbf{n}$ elementwise we have $f x=x$.

Altogether, we have now shown:

1.5 Theorem The homotopy groups of a symmetric spectrum are naturally tame $M$-modules. 
An example of an $M$-module which is not tame is the free module of rank 1. Tameness has many algebraic consequences which we discuss in the next section. We show in Remark 3.18 that we have now found all natural operations on the homotopy groups of a symmetric spectrum; more precisely, we show that the ring of natural operations on $\pi_{0} X$ is a completion of the monoid ring of $M$, so that an arbitrary operation is a sum, possibly infinite, of operations by elements from $M$.

The action of the monoid $M$ in used by Shipley in [7, Proposition 2.2.9], who credits this observation to Jeff Smith. To our knowledge, the tameness of this action, which is elementary but crucial for many things which we do in this paper, has not been exploited before.

1.6 Example To illustrate the action of the monoid $M$ on the homotopy groups of a symmetric spectrum $X$ we make it explicit for the injection $d: \omega \longrightarrow \omega$ given by $d(i)=i+1$, which will also play an important role later. For every $n \geq 1$, the map $d$ and the cycle $(1,2, \ldots, n, n+1)$ of $\Sigma_{n+1}$ agree on $\mathbf{n}$, so $d$ acts on $\pi_{k} X$ as the colimit of the system

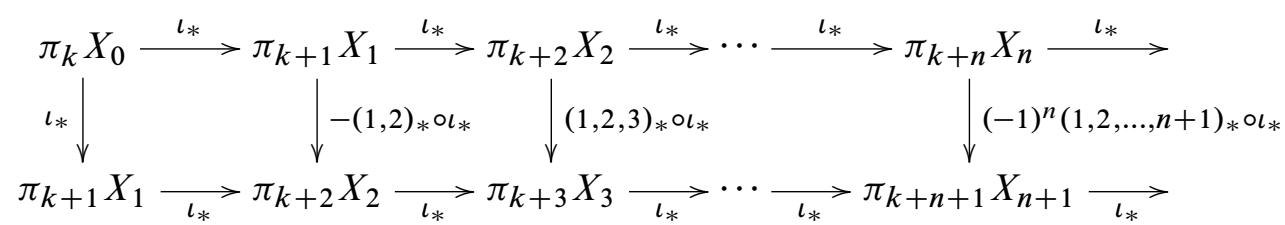

(at least for $k \geq 0$; for negative values of $k$ only a later portion of the system makes sense).

1.7 Remark The stable homotopy group $\pi_{k} X$ of a symmetric spectrum $X$ can also be calculated from the system of stable as opposed to unstable homotopy groups of the individual spaces $X_{n}$. For us, the $m$-th stable homotopy group $\pi_{m}^{\mathrm{s}} K$ of a pointed space $K$ is the colimit of the sequence of abelian groups

$$
\pi_{m} K \stackrel{S^{1} \wedge}{\longrightarrow} \pi_{1+m}\left(S^{1} \wedge K\right) \stackrel{S^{1} \wedge}{\longrightarrow} \pi_{2+m}\left(S^{2} \wedge K\right) \stackrel{S^{1} \wedge}{\longrightarrow} \cdots
$$

where we stabilize from the left. Smashing with the identity of $S^{1}$ from the right provides a stabilization map (even an isomorphism) $\pi_{m}^{\mathrm{s}} K \longrightarrow \pi_{m+1}^{\mathrm{s}}\left(K \wedge S^{1}\right)$. For a symmetric spectrum we can then define an $I$-functor $\underline{\pi}_{k}^{\mathrm{s}} X$ by setting $\left(\underline{\pi}_{k}^{\mathrm{s}} X\right)(\mathbf{n})=$ $\pi_{k+n}^{\mathrm{s}} X_{n}$ on objects (with no restriction on $k+n$ ) and defining the action of a morphism $\mathbf{n} \longrightarrow \mathbf{m}$ in the same way as for the $I$-functor $\underline{\pi}_{k} X$ of unstable homotopy groups.

The map from the initial term to the colimit of the sequence (1.8) provides a natural transformation $\pi_{m} K \longrightarrow \pi_{m}^{\mathrm{s}} K$ which is compatible with stabilization, so it defines 
a morphism of $I$-functors $\underline{\pi}_{k} X \longrightarrow \underline{\pi}_{k}^{\mathrm{s}} X$ for every symmetric spectrum $X$. The induced map on colimits

$$
\operatorname{colim}_{\mathbb{N}} \underline{\pi}_{k} X \stackrel{\cong}{\longrightarrow} \operatorname{colim}_{\mathbb{N}} \underline{\pi}_{k}^{\mathrm{s}} X
$$

is bijective (compare [7, Lemma 2.2.3]), thus an isomorphism of $M$-modules.

1.9 Remark We have chosen to let the spheres act from the right on the spaces in a symmetric spectrum, and not from the left as in [3]. The reason for this is that we find the $M$-action on the homotopy groups more transparent this way. More formally, we consider right $S$-modules in the category of symmetric sequences where Hovey, Shipley and Smith consider left modules. Since the sphere spectrum is a commutative monoid in symmetric sequences, its categories of left and right modules are isomorphic. However, the isomorphism is slightly more subtle than one may first think, and making consistent identifications of homotopy groups can be a little tricky. The reader is invited to translate our definition of the $M$-action on the homotopy groups of a right $S$-module via these isomorphism to left $S$-modules.

\section{Algebraic properties of tame $M$-modules}

In this section we discuss some algebraic properties of tame $M$-modules. It turns out that tameness is a rather restrictive condition. An example is that a tame $M$-module whose underlying abelian group is finitely generated must necessarily have a trivial $M$-action.

We introduce some useful notation and terminology. For an injective map $f: \omega \longrightarrow \omega$ we write $|f|$ for the smallest number $i \geq 0$ such that $f(i+1) \neq i+1$. So in particular, $f$ restricts to the identity on $\{1, \ldots,|f|\}$. We write $|\mathrm{Id}|=\infty$. An element $x$ of an $M$-module $W$ has filtration $n$ if for every $f \in M$ with $|f| \geq n$ we have $f x=x$. We denote by $W^{(n)}$ the subgroup of $W$ of elements of filtration $n$; for example, $W^{(0)}$ is the set of elements fixed by all $f \in M$. We say that $x$ has filtration exactly $n$ if it lies in $W^{(n)}$ but not in $W^{(n-1)}$. By definition, an $M$-module $W$ is tame if and only if every element has a finite filtration, ie, if the groups $W^{(n)}$ exhaust $W$.

The following lemmas collect some elementary observations, first for arbitrary $M-$ modules and then for tame $M$-modules.

2.1 Lemma Let $W$ be any $M$-module.

(i) If two elements $f$ and $g$ of $M$ coincide on $\mathbf{n}=\{1, \ldots, n\}$, then $f x=g x$ for all $x \in W$ of filtration $n$. 
(ii) For $n \geq 0$ and $f \in M$ set $m=\max \{f(\mathbf{n})\}$. Then $f \cdot W^{(n)} \subseteq W^{(m)}$.

(iii) We denote by $d \in M$ the map given by $d(i)=i+1$. If $x \in W$ has filtration exactly $n$ with $n \geq 1$, then $d x$ has filtration exactly $n+1$.

(iv) Let $V \subseteq W$ be an $M$-submodule such that the action of $M$ on $V$ and $W / V$ is trivial. Then the action of $M$ on $W$ is also trivial.

Proof (i) We can choose a bijection $\gamma \in M$ which agrees with $f$ and $g$ on $\mathbf{n}$, and then $\gamma^{-1} f$ and $\gamma^{-1} g$ fix $\mathbf{n}$ elementwise. So for $x$ of filtration $n$ we have $\left(\gamma^{-1} f\right) x=x=\left(\gamma^{-1} g\right) x$. Multiplying by $\gamma$ gives $f x=g x$.

(ii) If $g \in M$ satisfies $|g| \geq m$, then $g f$ and $f$ agree on $\mathbf{n}$. So for all $x \in W^{(n)}$ we have $g f x=f x$ by (i), which proves that $f x \in W^{(m)}$.

(iii) We have $d \cdot W^{(n)} \subseteq W^{(n+1)}$ by part (ii). To prove that $d$ increases the exact filtration we consider $x \in W^{(n)}$ with $n \geq 1$ and show that $d x \in W^{(n)}$ implies $x \in$ $W^{(n-1)}$.

For $f \in M$ with $|f|=n-1$ we define $g \in M$ by $g(1)=1$ and $g(i)=f(i-1)+1$ for $i \geq 2$. Then we have $g d=d f$ and $|g|=n$. We let $h$ be the cycle $h=(f(n)+$ $1, f(n), \ldots, 2,1)$ so that we have $|h d|=f(n)=\max \{f(\mathbf{n})\}$. Then $f x \in W^{(f(n))}$ by part (ii) and so

$$
f x=(h d)(f x)=h(g(d x))=(h d) x=x .
$$

Altogether this proves that $x \in W^{(n-1)}$.

(iv) Since the $M$-action is trivial on $V$ and $W / V$, every $f \in M$ determines an additive map $\delta_{f}: W / V \longrightarrow V$ such that $x-f x=\delta_{f}(x+V)$ for all $x \in W$. These maps satisfy $\delta_{f g}(x)=\delta_{f}(x)+\delta_{g}(x)$ and so $\delta$ is a homomorphism from the monoid $M$ to the abelian group of additive maps from $W / V$ to $V$. We will see in Lemma 5.2 below that the classifying space $B M$ of the monoid $M$ is contractible, so $H^{1}(B M, A)=$ $\operatorname{Hom}(M, A)$ is trivial for every abelian group $A$. Thus $\delta_{f}=0$ for all $f \in M$, ie, $M$ acts trivially on $W$.

2.2 Corollary The assignment $\mathbf{n} \mapsto W^{(n)}$ extends to an $I$-functor $W^{(\bullet)}$ in such a way that $W \mapsto W^{(\bullet)}$ is right adjoint to the functor which assigns to an $I$-functor $F$ the $M$-module $F(\omega)$. The counit of the adjunction $\left(W^{(\bullet)}\right)(\omega) \longrightarrow W$ is injective with image the subgroup of elements of finite filtration, which is also the largest tame submodule of $W$. The assignment $W \mapsto\left(W^{(\bullet)}\right)(\omega)=\bigcup_{n} W^{(n)}$ is right adjoint to the inclusion of tame $M$-modules into all $M$-modules. 
Proof To define the $I$-functor $W^{(\bullet)}$ on morphisms $\alpha: \mathbf{n} \longrightarrow \mathbf{m}$ in the category $I$ we choose any extension $\tilde{\alpha}: \omega \longrightarrow \omega$ of $\alpha$ and define $\alpha_{*}: W^{(n)} \longrightarrow W^{(m)}$ as the restriction of $\widetilde{\alpha} \cdot W \longrightarrow W$. This really has image in $W^{(m)}$ by part (ii) of Lemma 2.1 and is independent of the extension by (i) of that lemma. The rest is immediate.

2.3 Lemma Let $W$ be a tame $M$-module.

(i) Every element of $M$ acts injectively on $W$.

(ii) If the filtration of elements of $W$ is bounded, then $W$ is a trivial $M$-module.

(iii) If the map $d$ given by $d(i)=i+1$ acts surjectively on $W$, then $W$ is a trivial $M$-module.

(iv) If $W$ is finitely generated as an abelian group, then $W$ is a trivial $M$-module.

Proof (i) Consider $f \in M$ and $x \in W^{(n)}$ with $f x=0$. Since $f$ is injective, we can choose $h \in M$ with $|h f| \geq n$. Then $x=(h f) x=h(f x)=0$, so $f$ acts injectively.

(ii) Lemma 2.1 (iii) implies that if $W=W^{(n)}$ for some $n \geq 0$, then $n=0$, so the $M$-action is trivial.

(iii) Suppose $M$ does not act trivially, so that $W^{(0)} \neq W$. Let $n$ be the smallest positive integer such that $W^{(0)} \neq W^{(n)}$. Then by part (iii) of Lemma 2.1, any $x \in$ $W^{(n)}-W^{(0)}$ is not in the image of $d$, so $d$ does not act surjectively.

(iv) The union of the nested sequence of subgroups $W^{(0)} \subseteq W^{(1)} \subseteq W^{(2)} \subseteq \cdots$ is $W$. Since finitely generated abelian groups are noetherian, we have $W^{(n)}=W$ for all large enough $n$. By part (ii), the monoid $M$ must act trivially.

Parts (i), (iii) and (iv) of Lemma 2.3 can fail for nontame $M$-modules: we can let $f \in M$ act on the abelian group $\mathbb{Z}$ as the identity if the image of $f: \omega \longrightarrow \omega$ has finite complement, and we let $f$ acts as 0 if its image has infinite complement.

2.4 Example We introduce certain tame $M$-modules $\mathcal{P}_{n}$ for $n \geq 0$ which play important roles throughout this paper. The module $\mathcal{P}_{n}$ is the free abelian group with basis the set of ordered $n$-tuples of pairwise distinct elements of $\omega$ (or equivalently the set of injective maps from $\mathbf{n}$ to $\omega)$. The monoid $M$ acts from the left on this basis by componentwise evaluation, ie, $f\left(x_{1}, \ldots, x_{n}\right)=\left(f\left(x_{1}\right), \ldots, f\left(x_{n}\right)\right)$, and it acts on $\mathcal{P}_{n}$ by additive extension. For $n=0$, there is only one basis element, the empty tuple, and so $\mathcal{P}_{0}$ is isomorphic to $\mathbb{Z}$ with trivial $M$-action. For $n \geq 1$, the basis is countably infinite and the $M$-action is nontrivial. The module $\mathcal{P}_{n}$ is tame: the filtration of a basis element $\left(x_{1}, \ldots, x_{n}\right)$ is the maximum of the components. So the filtration subgroup 
$\mathcal{P}_{n}^{(m)}$ is generated by the $n$-tuples all of whose components are less than or equal to $m$. An equivalent way of saying this is that $\mathcal{P}_{n}^{(m)}=\mathbb{Z}[I(\mathbf{n}, \mathbf{m})]$, the free abelian group generated by all injections from $\mathbf{n}$ to $\mathbf{m}$; in particular, $\mathcal{P}_{n}^{(m)}$ is trivial for $m<n$.

The module $\mathcal{P}_{n}$ represents the functor of taking elements of filtration $n$ : for every $M$-module $W$, the map

$$
\operatorname{Hom}_{M-\bmod }\left(\mathcal{P}_{n}, W\right) \longrightarrow W^{(n)}, \quad \varphi \mapsto \varphi(1, \ldots, n)
$$

is bijective.

\section{Examples}

We discuss several classes of symmetric spectra with a view towards the $M$-action on the stable homotopy groups.

3.1 Example (Stabilizing homotopy groups) Let $X$ be a symmetric spectrum whose homotopy groups stabilize, ie, for each $k \in \mathbb{Z}$ there exists an $n \geq 0$ such that from the group $\pi_{k+n} X_{n}$ on, all maps in the system (1.1) defining $\pi_{k} X$ are isomorphisms. We claim that then the action of $M$ is trivial.

To prove the claim we consider more generally any $I$-functor $F$ for which there exists an $n \geq 0$ such that the natural map $F(\mathbf{n}) \longrightarrow F(\omega)$ to the colimit is surjective. This is certainly the case if from $\mathbf{n}$ on all maps $\iota_{*}$ in the colimit system are isomorphisms. In this situation every element of $F(\omega)$ has filtration $n$. But tame $M$-modules with bounded filtration necessarily have trivial $M$-action by Lemma 2.3 (ii).

Examples of symmetric spectra with stabilizing homotopy groups include all suspension spectra, $\Omega$-spectra, or $\Omega$-spectra from some point $X_{n}$ on. The symmetric spectrum obtained from a $\Gamma$-space [6] by evaluation on spheres is another example since for every $\Gamma$-space $A$ the structure map $A\left(S^{n}\right) \wedge S^{1} \longrightarrow A\left(S^{n+1}\right)$ is $(2 n+1)$-connected [4, Proposition 5.21].

3.2 Example (Orthogonal spectra) The monoid $M$ acts trivially on the homotopy group of every symmetric spectrum which is underlying an orthogonal spectrum [5, Example 4.4]. Indeed, the inclusion $\Sigma_{n} \longrightarrow O(n)$ as permutation matrices sends all even permutations to the path component of the unit in $O(n)$. So if the $\Sigma_{n}$-action on a pointed space $X_{n}$ extends to an $O(n)$-action, then all even permutations act as the identity on the homotopy groups of $X_{n}$.

So we consider more generally any $I$-functor $F$ which takes all even permutations to identity maps. Given $f \in M$ and an element $[x] \in F(\omega)$ in the colimit represented 
by $x \in F(\mathbf{n})$ we can find $m \geq \max \{f(\mathbf{n})\}$ and an even permutation $\gamma \in \Sigma_{m}$ such that $\gamma$ agrees with $f$ on $\mathbf{n}$. Since $\gamma$ is even, we then have $f_{*}[x]=\left[\left(\left.f\right|_{\mathbf{n}}\right)_{*}(x)\right]=$ $\left[(\gamma \mid \mathbf{n})_{*}(x)\right]=\left[\gamma_{*}\left(\iota_{*}^{m-n}(x)\right)\right]=\left[\iota_{*}^{m-n}(x)\right]=[x]$.

3.3 Example (Eilenberg-Mac Lane spectra) Every tame $M$-module $W$ can be realized as the homotopy group of a symmetric spectrum. For this purpose we modify the construction of the symmetric Eilenberg-Mac Lane spectrum of an abelian group, cf [3, Example 1.2.5]. We define

$$
(H W)_{n}=\left|W^{(n)} \otimes \mathbb{Z}\left[S^{n}\right]\right|,
$$

where $W^{(n)}$ is the filtration $n$ subgroup of $W, \mathbb{Z}\left[S^{n}\right]$ refers to the simplicial abelian group freely generated by the simplicial set $S^{n}=S^{1} \wedge \ldots \wedge S^{1}$, divided by the subgroup generated by the basepoint, and the bars denote geometric realization. The symmetric group $\Sigma_{n}$ takes $W^{(n)}$ to itself and we let it act diagonally on $(H W)_{n}$, ie, on $S^{n}$ by permuting the smash factors. The homotopy groups of the symmetric spectrum $H W$ are concentrated in dimension zero where we have $\pi_{0} H W \cong W$ as $M$-modules. If $M$ acts trivially on $W$, then this is just the ordinary Eilenberg-Mac Lane spectrum. (Instead of the system $\mathbf{n} \mapsto W^{(n)}$ we could use any $I$-functor in the definition above; this shows that every $I$-functor arises as the $I$-functor $\underline{\pi}_{0}$ of a symmetric spectrum).

3.4 Example (Free symmetric spectra) Hovey, Shipley and Smith observe in [3, Example 3.1.10] that the zeroth stable homotopy group of the free symmetric spectrum $F_{n} S^{n}$ is free abelian of countably infinite rank for $n \geq 1$. We refine this calculation to an isomorphism of $M$-modules $\pi_{0}\left(F_{n} S^{n}\right) \cong \mathcal{P}_{n}$; here $\mathcal{P}_{n}$ is the $M$-module which represents taking filtration $n$ elements; see Example 2.4. So while the groups $\pi_{0}\left(F_{n} S^{n}\right)$ are all additively isomorphic for different positive $n$, the $M$-action distinguishes them. In particular, there cannot be a chain of $\pi_{*}$-isomorphisms between $F_{n} S^{n}$ and $F_{m} S^{m}$ for $n \neq m$.

As in [3, Definition 2.2.5] we denote by $F_{n} K$ the free symmetric spectrum generated by a pointed space $K$ in level $n$. The free functor $F_{n}$ is left adjoint to evaluating a symmetric spectrum at level $n$.

We claim that there is a natural isomorphism of $M$-modules

$$
\pi_{k}\left(F_{n} K\right) \cong \mathcal{P}_{n} \otimes \pi_{k+n}^{\mathrm{s}} K .
$$

Here $\pi_{k+n}^{\mathrm{s}} K$ is the $(k+n)$-th stable homotopy group of $K$; the monoid $M$ acts only on $\mathcal{P}_{n}$. We postpone the proof to the next paragraph, where this isomorphism will be a special case of a more general statement. 
3.6 Example (Semifree symmetric spectra) Let $L$ be a pointed space with a basepoint preserving left action by the symmetric group $\Sigma_{n}$, for some $n \geq 0$. We let $H_{n} L$ denote the semifree symmetric spectrum generated by $L$, which we define as follows. First we let $L[n]$ denote the symmetric sequence which has the $\Sigma_{n}$-space $L$ in level $n$ and a point everywhere else, and then we set $H_{n} L=S \otimes L[n]$. The functor $H_{n}$ is left adjoint to evaluating a symmetric spectrum at level $n$, but now viewed as a functor with values in pointed $\Sigma_{n}$-spaces.

We construct a natural isomorphism of $M$-modules

$$
\pi_{k}\left(H_{n} L\right) \cong \mathcal{P}_{n} \otimes_{\Sigma_{n}}\left(\pi_{k+n}^{\mathrm{s}} L\right)(\mathrm{sgn})
$$

Here we use the right $\Sigma_{n}$-action on the tame $M$-module $\mathcal{P}_{n}$ given on the basis by permuting the components of an $n$-tuple, ie, $\left(x_{1}, \ldots, x_{n}\right) \gamma=\left(x_{\gamma(1)}, \ldots, x_{\gamma(n)}\right)$. On the right of the tensor symbol, the group $\Sigma_{n}$ acts by what is induced on stable homotopy groups by the action on $L$, twisted by sign. For a pointed space $K$ (without any group action) we have $H_{n}\left(\Sigma_{n}^{+} \wedge K\right) \cong F_{n} K$, which makes the isomorphism (3.5) a special case of (3.7).

The construction of the isomorphism (3.7) starts from the more explicit description of the semifree spectrum as

$$
\left(H_{n} L\right)_{n+m}=\Sigma_{n+m}^{+} \wedge \Sigma_{n} \times \Sigma_{m}\left(L \wedge S^{m}\right)
$$

( $H_{n} L$ consists only of a point in levels less than $n$ ). The structure map

$$
\sigma_{n+m}:\left(\Sigma_{n+m}^{+} \wedge \Sigma_{n} \times \Sigma_{m}\left(L \wedge S^{m}\right)\right) \wedge S^{1} \longrightarrow \Sigma_{n+m+1}^{+} \wedge \Sigma_{n} \times \Sigma_{m+1}\left(L \wedge S^{m+1}\right)
$$

arises from the inclusion $\Sigma_{n+m} \longrightarrow \Sigma_{n+m+1}$ (as the subgroup fixing the element $n+m+1)$ and the identification $S^{m} \wedge S^{1} \cong S^{m+1}$.

We calculate the $I$-functor $\underline{\pi}_{k}^{\mathrm{s}}\left(H_{n} L\right)$ consisting of the stable homotopy groups of the spaces $\left(H_{n} L\right)_{k+n}$ and exploit that for any symmetric spectrum $X$ the $M$-module $\pi_{k} X$ can also be calculated as the colimit of $\underline{\pi}_{k}^{\mathrm{s}} X$ instead of the $I$-functor $\underline{\pi}_{k} X$ of unstable homotopy groups; see Remark 1.7. We start with the isomorphism of $\Sigma_{n+m}$-modules

$$
\begin{aligned}
\pi_{k+n+m}^{\mathrm{s}}\left(H_{n} L\right)_{n+m} & =\pi_{k+n+m}^{\mathrm{s}}\left(\Sigma_{n+m}^{+} \wedge \Sigma_{n} \times \Sigma_{m}\left(L \wedge S^{m}\right)\right) \\
& \cong \mathbb{Z}\left[\Sigma_{n+m}\right] \otimes_{\mathbb{Z}\left[\Sigma_{n} \times \Sigma_{m}\right]}\left(\pi_{k+n}^{\mathrm{s}} L \otimes \operatorname{sgn}_{m}\right) .
\end{aligned}
$$


The coordinate permutations of $S^{m}$ act by sign on stable homotopy groups, whence the sign representation $\operatorname{sgn}_{m}$ of $\Sigma_{m}$. For any $\Sigma_{n}$-module $B$ the map

$$
\begin{aligned}
\mathbb{Z}\left[\Sigma_{n+m}\right] \otimes_{\mathbb{Z}\left[\Sigma_{n} \times \Sigma_{m}\right]}\left(B \otimes \operatorname{sgn}_{m}\right) & \longrightarrow \mathcal{P}_{n}^{(n+m)} \otimes_{\Sigma_{n}} B(\operatorname{sgn}) \\
\gamma \otimes(b \otimes 1) & \longmapsto \operatorname{sgn}(\gamma) \cdot(\gamma(1), \ldots, \gamma(n)) \otimes b
\end{aligned}
$$

is an isomorphism of $\Sigma_{n+m}$-modules, where $\mathcal{P}_{n}^{(n+m)}$ is the filtration $n+m$ subgroup of the $M$-module $\mathcal{P}_{n}$. Taking $B=\pi_{k+n}^{\mathrm{s}} L$ and combining all the above yields isomorphisms of $\Sigma_{n+m}$-modules

$$
\pi_{k+n+m}^{\mathrm{s}}\left(H_{n} L\right)_{n+m} \cong \mathcal{P}_{n}^{(n+m)} \otimes_{\Sigma_{n}}\left(\pi_{k+n}^{\mathrm{s}} L\right)(\mathrm{sgn})
$$

which as $n+m$ varies constitute an isomorphism of $I$-functors

$$
\underline{\pi}_{k}^{\mathrm{s}}\left(H_{n} L\right) \cong \mathcal{P}_{n}^{(-)} \otimes_{\Sigma_{n}}\left(\pi_{k+n}^{\mathrm{s}} L\right)(\mathrm{sgn}) .
$$

Taking colimits gives the isomorphism of $M$-modules (3.7).

3.8 Example (Infinite products) Finite products of symmetric spectra are $\pi_{*}$-isomorphic to finite wedges, so stable homotopy groups commute with finite products. But homotopy groups do not in general commute with infinite products. This should not be surprising because stable homotopy groups involve a sequential colimit, and these generally do not preserve infinite products.

There are even two different ways in which commutation with products can fail. First we note that an infinite product of a family $\left\{W_{i}\right\}_{i \in I}$ of tame $M$-modules is only tame if almost all the modules $W_{i}$ have trivial $M$-action. Indeed, if there are infinitely many $W_{i}$ with nontrivial $M$-action, then by Lemma 2.3 (ii) the product $\prod_{i \in I} W_{i}$ contains tuples of elements whose filtrations are not bounded. We define the tame product of the family $\left\{W_{i}\right\}_{i \in I}$ by

$$
\prod_{i \in I}^{\text {tame }} W_{i}=\bigcup_{n \geq 0}\left(\prod_{i \in I} W_{i}^{(n)}\right),
$$

which is the largest tame submodule of the product and thus the categorical product in the category of tame $M$-modules.

Now we consider a family $\left\{X_{i}\right\}_{i \in I}$ of symmetric spectra. Since the monoid $M$ acts tamely on the homotopy groups of any symmetric spectrum, the natural map from the homotopy groups of the product spectrum to the product of the homotopy groups always lands in the tame product. But in general, this natural map

$$
\pi_{k}\left(\prod_{i \in I} X_{i}\right) \longrightarrow \prod_{i \in I}^{\text {tame }} \pi_{k} X_{i}
$$


need not be an isomorphism. As an example we consider the symmetric spectra $\left(F_{1} S^{1}\right)^{\leq i}$ obtained by truncating the free symmetric spectrum $F_{1} S^{1}$ above level $i$, ie,

$$
\left(\left(F_{1} S^{1}\right)^{\leq i}\right)_{n}=\left\{\begin{array}{cl}
\left(F_{1} S^{1}\right)_{n} & \text { for } n \leq i, \\
* & \text { for } n \geq i+1
\end{array}\right.
$$

with structure maps as a quotient spectrum of $F_{1} S^{1}$. Then $\left(F_{1} S^{1}\right)^{\leq i}$ has trivial homotopy groups for all $i$. The 0 -th homotopy group of the product $\prod_{i \geq 1}\left(F_{1} S^{1}\right)^{\leq i}$ is the colimit of the sequence of maps

$$
\prod_{i \geq n} \mathcal{P}_{1}^{(n)} \longrightarrow \prod_{i \geq n+1} \mathcal{P}_{1}^{(n+1)}
$$

which first projects away from the factor indexed by $i=n$ and then takes a product of inclusions $\mathcal{P}_{1}^{(n)} \longrightarrow \mathcal{P}_{1}^{(n+1)}$. The colimit is the quotient of the tame product $\prod_{i \geq 1}^{\text {tame }} \mathcal{P}_{1}$ by the sum $\bigoplus_{i \geq 1} \mathcal{P}_{1}$; so $\pi_{0}$ of the product is nonzero and even has a nontrivial $M$-action.

3.10 Example (Loop and suspension) The loop $\Omega X$ and suspension $S^{1} \wedge X$ of a symmetric spectrum $X$ are defined by applying the functors $\Omega$ respectively $S^{1} \wedge-$ levelwise, where the structure maps do not interact with the new loop or suspension coordinates. We claim that loop and suspension simply shift the homotopy groups while leaving the $M$-action unchanged.

We use the isomorphism $\alpha: \pi_{k+n} \Omega\left(X_{n}\right) \cong \pi_{1+k+n} X_{n}$ defined by sending a representing continuous map $f: S^{k+n} \longrightarrow \Omega\left(X_{n}\right)$ to the class of the adjoint $\hat{f}: S^{1+k+n} \longrightarrow X_{n}$ given by $\hat{f}(s \wedge t)=f(t)(s)$, where $s \in S^{1}, t \in S^{k+n}$. As $n$ varies, these particular isomorphisms are compatible with the symmetric group actions and stabilization maps, so they form an isomorphism of $I$-functors $\alpha: \underline{\pi}_{k}(\Omega X) \cong \underline{\pi}_{1+k} X$, hence induce an isomorphism of $M$-modules

$$
\alpha: \pi_{k}(\Omega X) \stackrel{\cong}{\longrightarrow} \pi_{1+k} X
$$

on colimits.

For every symmetric spectrum $X$ the map $S^{1} \wedge-: \pi_{k+n} X_{n} \longrightarrow \pi_{1+k+n}\left(S^{1} \wedge X_{n}\right)$ is $\Sigma_{n}$-equivariant and a natural transformations of $I$-functors as $n$ varies. So it induces a natural map

$$
\beta: \pi_{k} X \longrightarrow \pi_{1+k}\left(S^{1} \wedge X\right)
$$

which is $M$-linear and an isomorphism by "stable excision"; see [3, Lemma 3.1.13].

Moreover, the composite

$$
\pi_{*} X \stackrel{\beta}{\longrightarrow} \pi_{1+*}\left(S^{1} \wedge X\right) \stackrel{\alpha^{-1}}{\longrightarrow} \pi_{*}\left(\Omega\left(S^{1} \wedge X\right)\right)
$$


is the map induced by the adjunction unit $X \longrightarrow \Omega\left(S^{1} \wedge X\right)$ on homotopy.

3.11 Example (Shift) The shift is another construction for symmetric spectra which reindexes the homotopy groups, but unlike the suspension, this construction changes the $M$-action in a systematic way. The shift of a symmetric spectrum $X$ is given by

$$
(\operatorname{sh} X)_{n}=X_{1+n}
$$

with action of $\Sigma_{n}$ via the monomorphism $(1 \times-): \Sigma_{n} \longrightarrow \Sigma_{1+n}$ which is explicitly given by $(1 \times \gamma)(1)=1$ and $(1 \times \gamma)(i)=\gamma(i-1)+1$ for $2 \leq i \leq 1+n$. The structure maps of $\operatorname{sh} X$ are the reindexed structure maps for $X$. An isomorphic description of the shift is as a symmetric function spectrum [3, Definition 2.2.9]

$$
\operatorname{sh} X=\operatorname{Hom}_{S}\left(F_{1} S^{0}, X\right),
$$

which shows that the shift functor has a left adjoint given by smashing with $F_{1} S^{0}$.

If we view $\Sigma_{n}$ as the subgroup of $M$ of maps which fix all numbers bigger than $n$, then the homomorphism $(1 \times-): \Sigma_{n} \longrightarrow \Sigma_{1+n}$ has a natural extension to a monomorphism $(1 \times-): M \longrightarrow M$ given by $(1 \times f)(1)=1$ and $(1 \times f)(i)=f(i-1)+1$ for $i \geq 2$. The image of the monomorphism $1 \times-$ is the submonoid of those $g \in M$ with $g(1)=1$. If $W$ is an $M$-module, we denote by $W(1)$ the $M$-module with the same underlying abelian group, but with $M$-action through the endomorphism $1 \times-$. We call $W(1)$ the shift of $W$. Since $|1 \times f|=1+|f|$, shifting an $M$-module shifts the filtration subgroups, ie, we have $M(1)^{(n)}=M^{(1+n)}$ for all $n \geq 0$. Thus the $M$-module $W(1)$ is tame if and only if $W$ is.

For any symmetric spectrum $X$, integer $k$ and large enough $n$ we have

$$
\pi_{(k+1)+n}(\operatorname{sh} X)_{n}=\pi_{k+(1+n)} X_{1+n},
$$

and the maps in the colimit system for $\pi_{k+1}(\operatorname{sh} X)$ are the same as the maps in the colimit system for $\pi_{k} X$. Thus we get $\pi_{k+1}(\operatorname{sh} X)=\pi_{k} X$ as abelian groups. However, the action of a permutation on $\pi_{k+1+n}(\operatorname{sh} X)_{n}$ is shifted by the homomorphism $1 \times-$, so we have

$$
\pi_{*+1}(\operatorname{sh} X)=\left(\pi_{*} X\right)(1)
$$

as $M$-modules.

Shifting preserves $\pi_{*}$-isomorphisms because of (3.12), but shifting does not in general preserve stable equivalences of symmetric spectra. An example is the fundamental stable equivalence $\lambda: F_{1} S^{1} \longrightarrow S$ of [3, Example 3.1.10] which is adjoint to the identity of $S^{1}$. The symmetric spectrum $\operatorname{sh}\left(F_{1} S^{1}\right)$ is isomorphic to the wedge of $F_{0} S^{1}$ and 
$F_{1} S^{2}$, while $\operatorname{sh} S \cong F_{0} S^{1}$; the map $\operatorname{sh} \lambda: \operatorname{sh}\left(F_{1} S^{1}\right) \longrightarrow \operatorname{sh} S$ is the projection to a wedge summand whose complementary summand $F_{1} S^{2}$ is not stably contractible.

3.13 Example (Shift adjoint) We calculate the effect on homotopy groups of the left adjoint of shifting by establishing a natural isomorphism of $M$-modules

$$
\pi_{k}\left(F_{1} S^{0} \wedge X\right) \cong \mathbb{Z}[M]^{+} \otimes_{\mathbb{Z}[M]} \pi_{k+1} X .
$$

Here $\mathbb{Z}[M]^{+}$denotes the monoid ring of $M$ with its usual left action, but with right action through the monomorphism $(1 \times-): M \longrightarrow M$ given by $(1 \times f)(1)=1$ and $(1 \times f)(i)=f(i-1)+1$ for $i \geq 2$. As a right $M$-module, $\mathbb{Z}[M]^{+}$is free of countably infinite rank (one possible basis is given by the transpositions $(1, n)$ for $n \geq 1$ ). So the isomorphism (3.14) in particular implies that the underlying abelian group of $\pi_{k}\left(F_{1} S^{0} \wedge X\right)$ is a countably infinite sum of copies of the underlying abelian group of $\pi_{k+1} X$.

The spectrum $F_{1} S^{0} \wedge Y$ is trivial in level 0 and in positive levels we have

$$
\left(F_{1} S^{0} \wedge X\right)_{1+n}=\Sigma_{1+n}^{+} \wedge_{\Sigma_{n}} X_{n} .
$$

Here $\Sigma_{n}$ acts from the right on $\Sigma_{1+n}$ via the monomorphism $(1 \times-): \Sigma_{n} \longrightarrow \Sigma_{1+n}$. The structure map $\left(\Sigma_{1+n}^{+} \wedge \Sigma_{n} X_{n}\right) \wedge S^{1} \longrightarrow \Sigma_{1+n+1}^{+} \wedge \Sigma_{n+1} X_{n+1}$ is induced by $(-\times 1): \Sigma_{1+n} \longrightarrow \Sigma_{1+n+1}$ (the "inclusion") and the structure map of $X$. Taking stable homotopy groups we get a $\Sigma_{1+n}$-equivariant isomorphism

$$
\begin{aligned}
\pi_{k+(1+n)}^{\mathrm{s}}\left(F_{1} S^{0} \wedge X\right)_{1+n} & =\pi_{k+1+n}^{\mathrm{s}}\left(\Sigma_{1+n}^{+} \wedge_{\Sigma_{n}} X_{n}\right) \\
& \cong \mathbb{Z}\left[\Sigma_{1+n}\right] \otimes_{\mathbb{Z}\left[\Sigma_{n}\right]} \pi_{(k+1)+n}^{\mathrm{s}} X_{n} .
\end{aligned}
$$

Taking the colimit over the stabilization maps gives an isomorphism

$$
\pi_{k}\left(F_{1} S^{0} \wedge X\right) \cong \mathbb{Z}\left[\Sigma_{\infty}\right]^{+} \otimes_{\mathbb{Z}\left[\Sigma_{\infty}\right]} \pi_{k+1} X
$$

where $\Sigma_{\infty}$ is the subgroup of $M$ consisting of bijections which fix almost all elements of $\omega$. The isomorphism (3.14) is then obtained from the observation that for every tame $M$-module $W$, the natural map $\mathbb{Z}\left[\Sigma_{\infty}\right]^{+} \otimes_{\mathbb{Z}\left[\Sigma_{\infty}\right]} W \longrightarrow \mathbb{Z}[M]^{+} \otimes_{\mathbb{Z}[M]} W$ is a bijection.

We note that the functor $\mathbb{Z}[M]^{+} \otimes_{\mathbb{Z}[M]}-$ is left adjoint to $\operatorname{Hom}_{M}\left(\mathbb{Z}[M]^{+},-\right)$, which is a fancy way of writing the algebraic shift functor $W \mapsto W(1)$. Under the isomorphism (3.14) and the identification (3.12), the adjunction between $\operatorname{sh}=\operatorname{Hom}_{S}\left(F_{1} S^{0},-\right)$ and $F_{1} S^{0} \wedge-$ as functors of symmetric spectra corresponds exactly to the adjunction between $W \mapsto W(1)$ and $\mathbb{Z}[M]^{+} \otimes_{\mathbb{Z}[M]}-$ as functors of tame $M$-modules. 
3.15 Example (Homotopy of $R^{\infty} X$ ) In the proof of [3, Theorem 3.1.11], Hovey, Shipley and Smith define spectra $R X$ and $R^{\infty} X$ which come up when studying the relationship between $\pi_{*}$-isomorphisms and stable equivalences and which reappear in the characterization of semistable symmetric spectra in [3, Proposition 5.6.2]. We exhibit a natural isomorphism of $M$-modules

$$
\pi_{k}\left(R^{\infty} X\right) \cong\left(\pi_{k} X\right)(\infty) .
$$

Here for an $M$-module $V$ we denote by $V(\infty)$ the colimit of the sequence

$$
V \stackrel{d}{\longrightarrow} V(1) \stackrel{d \cdot}{\longrightarrow} V(2) \stackrel{d \cdot}{\longrightarrow} \cdots
$$

(note that $(1 \times f) d=d f$ for all $f \in M$, which means that $d \cdot V \longrightarrow V(1)$ is indeed $M$-linear, and so the colimit $V(\infty)$ is naturally an $M$-module).

By definition we have $R X=\operatorname{Hom}_{S}\left(F_{1} S^{1}, X\right)=\Omega(\operatorname{sh} X)$. Let $\lambda: F_{1} S^{1} \longrightarrow S$ denote the morphism adjoint to the identity of $S^{1}$; this is the prototype of a stable equivalence which is not a $\pi_{*}$-isomorphism; compare [3, Example 3.1.10]. Then $\lambda$ induces a morphism on function spectra

$$
\lambda^{*}: X=\operatorname{Hom}_{S}(S, X) \longrightarrow \operatorname{Hom}_{S}\left(F_{1} S^{1}, X\right)=R X
$$

and $R^{\infty} X$ is the colimit of the sequence

$$
X \stackrel{\lambda^{*}}{\longrightarrow} R X \stackrel{R\left(\lambda^{*}\right)}{\longrightarrow} R^{2} X \stackrel{R^{2}\left(\lambda^{*}\right)}{\longrightarrow} \cdots .
$$

For calculating the homotopy of $R^{\infty} X$ we identify the effect of $\lambda^{*}: X \longrightarrow R X=$ $\Omega(\operatorname{sh} X)$ in homotopy. The level $n$ component $\lambda_{n}^{*}: X_{n} \longrightarrow(R X)_{n}=\Omega\left(X_{1+n}\right)$ is adjoint to the composite

$$
S^{1} \wedge X_{n} \underset{\text { twist }}{\stackrel{\cong}{\longrightarrow}} X_{n} \wedge S^{1} \stackrel{\sigma_{n}}{\longrightarrow} X_{n+1} \stackrel{(1, \ldots, n, n+1)}{\longrightarrow} X_{1+n}
$$

(using only the structure map $\sigma_{n}$ without the twist isomorphism and cycle permutation $(1, \ldots, n, n+1)$ does not yield a morphism of symmetric spectra!) So the square

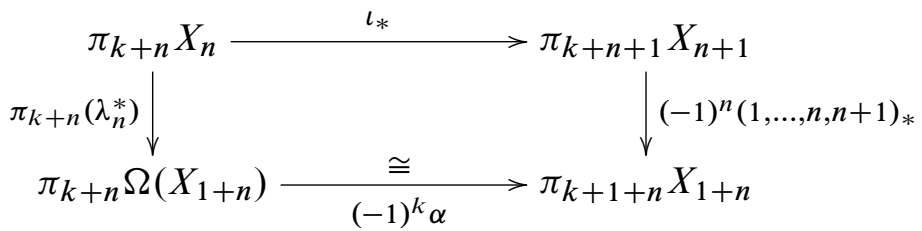

commutes, where $\iota_{*}$ is the stabilization map, and the isomorphism $\alpha$ is as in Example 3.10. The signs arise as the effect of moving a sphere coordinate past $k$ respectively $n$ 
other coordinates. As $n$ increases, the maps

$$
(-1)^{n}(1, \ldots, n, n+1)_{*} \circ \iota_{*}: \pi_{k+n} X_{n} \longrightarrow \pi_{k+1+n} X_{1+n}
$$

stabilize to the left multiplication of $d \in M$ on $\pi_{k} X$; see Example 1.6. So we have shown that the square

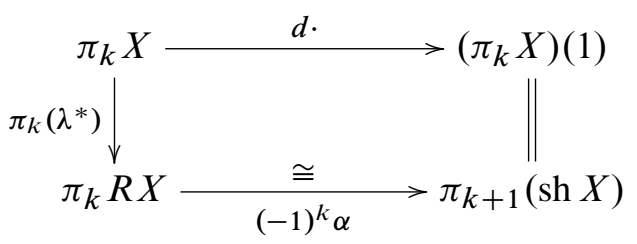

commutes. If we iterate applications of $R$ and pass to the colimit, we obtain the isomorphism (3.16).

3.18 Remark The monoid $M$ gives essentially all natural operations on the homotopy groups of symmetric spectra. More precisely, we now identify the ring of natural operations $\pi_{0} X \longrightarrow \pi_{0} X$ with a completion of the monoid ring $\mathbb{Z}[M]$. Moreover, tame $M$-modules can equivalently be described as the discrete modules over the ring of operations. We will not need this information later, so we will be brief.

We define the ring $\mathbb{Z}[[M]]$ as the endomorphism ring of the functor $\pi_{0}: S p^{\Sigma} \longrightarrow$ $\mathcal{A} b$. So an element of $\mathbb{Z}[[M]]$ is a natural self-transformation of the functor $\pi_{0}$, and composition of transformations gives the product. The following calculation of this ring depends on the fact that the homotopy group functor $\pi_{0}$ is prorepresented, in the level homotopy category of symmetric spectra, by the inverse system of free symmetric spectra $F_{n} S^{n}$, and that we know $\pi_{0}\left(F_{n} S^{n}\right)$ by Example 3.4.

In more detail: for every $n \geq 0$ we let $j_{n} \in \pi_{n}\left(F_{n} S^{n}\right)_{n}$ be the wedge summand inclusion $S^{n} \longrightarrow \Sigma_{n}^{+} \wedge S^{n}=\left(F_{n} S^{n}\right)_{n}$ indexed by the unit element of $\Sigma_{n}$. Then evaluation at $j_{n}$ is a bijection

$$
\left[F_{n} S^{n}, X\right] \longrightarrow \pi_{n} X_{n}, \quad[f] \mapsto f_{*}\left(j_{n}\right)
$$

where the left hand side means homotopy classes of morphisms of symmetric spectra. We write $\lambda: F_{n+1} S^{n+1} \longrightarrow F_{n} S^{n}$ for the morphism adjoint the wedge summand inclusion $S^{n+1} \longrightarrow \Sigma_{n+1}^{+} \wedge\left(S^{n} \wedge S^{1}\right)=\left(F_{n} S^{n}\right)_{n+1}$ indexed by the unit element of $\Sigma_{n+1}$. Then we have

$$
\lambda_{*}\left(j_{n+1}\right)=\iota_{*}\left(j_{n}\right)
$$


in the group $\pi_{n+1}\left(F_{n} S^{n}\right)_{n+1}$ which implies that the squares

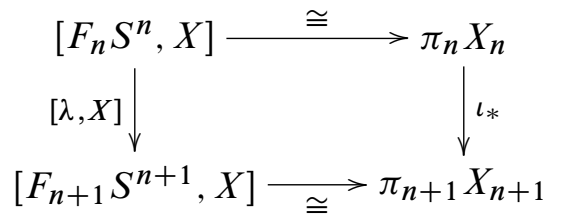

commute. Passage to colimits gives a natural isomorphism

$$
\operatorname{colim}_{n}\left[F_{n} S^{n}, X\right] \longrightarrow \pi_{0} X .
$$

From here the Yoneda lemma shows that we get an isomorphism of abelian groups

$$
\beta: \mathbb{Z}[[M]] \longrightarrow \lim _{n} \pi_{0}\left(F_{n} S^{n}\right)
$$

(where the limit is taken over the maps $\pi_{0} \lambda$ ) by sending a natural transformation $\tau: \pi_{0} \longrightarrow \pi_{0}$ to the tuple $\left\{\tau_{F_{n} S^{n}}\left[j_{n}\right]\right\}_{n}$.

It remains to exhibit the ring $\mathbb{Z}[[M]]$ as a completion of the monoid ring $\mathbb{Z}[M]$. The natural action of $M$ on the 0 -th homotopy group of a symmetric spectrum provides a ring homomorphism $\mathbb{Z}[M] \longrightarrow \mathbb{Z}[[M]]$. We define a left ideal $I_{n}$ of $\mathbb{Z}[M]$ as the subgroup generated by all differences of the form $f-g$ for all $f, g \in M$ such that $f$ and $g$ agree on $\mathbf{n}$. If $W$ is a tame $M$-module and if $x \in W^{(n)}$ has filtration $n$, then $I_{n} \cdot x=0$. So the action of the monoid ring $\mathbb{Z}[M]$ on any tame module automatically extends to an additive map

$$
\left(\lim _{n} \mathbb{Z}[M] / I_{n}\right) \otimes W \longrightarrow W .
$$

(Warning: $I_{n}$ is not a right ideal for $n \geq 1$, so the completion does not a priori have a ring structure). Since the homotopy groups of every symmetric spectrum form tame $M$-modules, this gives a map of abelian groups

$$
\alpha: \lim _{n} \mathbb{Z}[M] / I_{n} \longrightarrow \mathbb{Z}[[M]]
$$

which extends the map from the monoid ring $\mathbb{Z}[M]$.

To prove that $\alpha$ is a bijection we show that the composite $\beta \alpha: \lim _{n} \mathbb{Z}[M] / I_{n} \longrightarrow$ $\lim _{n} \pi_{0}\left(F_{n} S^{n}\right)$ with the isomorphism (3.19) is bijective. But this holds because the composite arises from compatible isomorphisms

$$
\mathbb{Z}[M] / I_{n} \longrightarrow \pi_{0}\left(F_{n} S^{n}\right), \quad f+I_{n} \longmapsto f \cdot\left[j_{n}\right],
$$

which in turn uses the isomorphisms $\mathcal{P}_{n} \cong \pi_{0}\left(F_{n} S^{n}\right)$ from Example 3.4. 
We end this remark by claiming without proof that the extended action of $\mathbb{Z}[[M]]$ on a tame $M$-module $W$ makes it a discrete module in the sense that the action map

$$
\mathbb{Z}[[M]] \times W \longrightarrow W
$$

is continuous with respect to the discrete topology on $W$ and the filtration topology on $\mathbb{Z}[[M]]$. Conversely, if $W$ is a discrete module over $\mathbb{Z}[[M]]$, then its underlying $M$-module is tame. This establishes an isomorphism between the category of tame $M$-modules and the category of discrete $\mathbb{Z}[[M]]$-modules.

\section{Semistable symmetric spectra}

The semistable spectra form an important class of symmetric spectra since between these, stable equivalences coincide with $\pi_{*}$-isomorphisms. The reason behind this is that for semistable spectra, the naively defined homotopy groups of (1.1) coincide with the "true" homotopy groups, ie, morphisms in the stable homotopy category from the sphere spectra.

More formally, a symmetric spectrum is semistable in the sense of [3, Definition 5.6.1] if some (hence any) stably fibrant replacement is a $\pi_{*}$-isomorphism. Here a stably fibrant replacement is a stable equivalence $X \longrightarrow L X$ with target an $\Omega$-spectrum. Many symmetric spectra which arise naturally are semistable (compare Example 4.2), and Section 5.6 of [3] gives some criteria for checking semistability. We now provide a criterion for semistability in terms of the $M$-action on the homotopy groups of a symmetric spectra.

4.1 Theorem A symmetric spectrum is semistable if and only if the $M$-action on all of its homotopy groups is trivial.

Proof By [3, Proposition 5.6.2], $X$ is semistable if and only if the map $\lambda^{*}: X \longrightarrow$ $R X=\Omega(\operatorname{sh} X)$ is a $\pi_{*}$-isomorphism (since we work with topological spaces and not simplicial sets, we do not need any level fibrant replacement). By (3.17) this happens if and only if left multiplication by $d$ is an isomorphism on all homotopy groups, which by Lemma 2.3 (iii) is equivalent to a trivial $M$-action.

The "trivial $M$-action" criterion is often handy for deciding about semistability and for showing that semistability is preserved by certain constructions. We give a few examples of this: 
4.2 Example By Example 3.1 any symmetric spectrum whose homotopy groups stabilize has trivial $M$-action and is thus semistable (compare Proposition 5.6 .4 (2) of [3]). This applies in particular to suspension spectra, $\Omega$-spectra (possibly only from some later point on), or symmetric spectra associated to $\Gamma$-spaces. By Example 3.2, the underlying symmetric spectrum of every orthogonal spectrum has trivial $M$-action on homotopy groups, and is thus semistable.

4.3 Example Let $X$ be a symmetric spectrum whose homotopy groups are dimensionwise finitely generated as abelian groups. Tameness then forces the $M$-action to be trivial (Lemma 2.3 (iv)) and so $X$ is semistable. This is a strengthening of Proposition 5.6.4 (1) of [3], where is it is proved that spectra with finite homotopy groups are semistable.

4.4 Example Example 3.8 shows that an infinite product of symmetric spectra with trivial homotopy groups can have homotopy groups with nontrivial $M$-action. In particular, infinite products of semistable symmetric spectra need not be semistable.

4.5 Example If $f: X \longrightarrow Y$ is any morphism of symmetric spectra, then the homotopy groups of the spectra $X, Y$ and the mapping cone $C(f)=[0,1]^{+} \wedge X \cup_{f} Y$ are related by a long exact sequence of tame $M$-modules (see [5, Thm 7.4 (vi)]; we use that the $M$-action does not change under loop and suspension). Trivial tame $M$-modules are closed under taking submodules, quotient modules and extensions (Lemma 2.1 (iv)); so if two out of the three graded $M$-modules $\pi_{*} X, \pi_{*} Y$ and $\pi_{*} C(f)$ have trivial $M$-action, then so does the third. Thus the mapping cone of any morphism between semistable symmetric spectra is semistable.

If $f: X \longrightarrow Y$ is an h-cofibration [5, Section 5] of symmetric spectra, or simply an injective morphism when in the simplicial context of [3], then the mapping cone $C(f)$ is $\pi_{*}$-isomorphic to the quotient $Y / X$. Thus if two of the spectra $X, Y$ and $Y / X$ are semistable, then so is the third.

4.6 Example Semistability is preserved under suspension, loop, wedges, shift and sequential colimits along h-cofibrations (or injective morphisms when in the simplicial context) since these operations preserve the property of $M$ acting trivially on homotopy groups.

4.7 Example For a symmetric spectrum $X$ and a pointed space $K$, let $K \wedge X$ be the symmetric spectrum obtained by smashing $K$ levelwise with $X$. So $(K \wedge X)_{n}=$ $K \wedge X_{n}$, with $\Sigma_{n}$-action by the given action on $X_{n}$, and with structure map Id $\wedge \sigma_{n}: K \wedge X_{n} \wedge S^{1} \longrightarrow K \wedge X_{n+1}$. For example, when $K=S^{1}$ is the circle, 
this specializes to the suspension of $X$. We claim that if $X$ is semistable and $K$ is a $\mathrm{CW}$-complex, then the symmetric spectrum $K \wedge X$ is again semistable.

We first prove the claim for finite dimensional $\mathrm{CW}$-complexes by induction over the dimension. If $K$ is 0 -dimensional, then $K \wedge X$ is a wedge of copies of $X$, thus semistable. If $K$ has positive dimension $n$ and $K_{(n-1)}$ is its $(n-1)$-skeleton, then $K / K_{(n-1)}$ is a wedge of $n$-spheres and so the quotient of $K \wedge X$ by the subspectrum $K_{(n-1)} \wedge X$ is a wedge of $n$-fold suspension of $X$. By induction the subspectrum $K_{(n-1)} \wedge X$ is semistable; since the inclusion is an h-cofibration and the quotient spectrum is also semistable, so is $K \wedge X$. For a general CW-complex $K$ the symmetric spectrum $K \wedge X$ is the sequential colimit, over h-cofibrations, of the smash product of $X$ with the skeleta of $K$. So $K \wedge X$ is semistable.

The geometric realization of any simplicial set is a CW-complex, so in the simplicial context of [3] we deduce that for any pointed simplicial set $K$ and any semistable symmetric spectrum $X$ the symmetric spectrum $K \wedge X$ is again semistable.

4.8 Example Let $F: J \longrightarrow S p^{\Sigma}$ be a functor from a small category $J$ to the category of symmetric spectra. If $F(j)$ is semistable for each object $j$ of $J$, then the homotopy colimit of $F$ over $J$ is semistable.

Indeed, the homotopy colimit is the geometric realization of the simplicial replacement $\amalg_{*} F$ in the sense of Bousfield and Kan [2, Chapter XII, 5.1], a simplicial object of symmetric spectra. The spectrum of $n$-simplices of $\amalg_{*} F$ is a wedge, indexed over the $n$-simplices of the nerve of $J$, of spectra which occur as values of $F$. The geometric realization $\left|\amalg_{*} F\right|$ is the sequential colimit, over h-cofibrations, of the realizations of the skeleta $\mathrm{sk}_{n} \amalg_{*} F$ in the simplicial direction, so it suffices to show that each of these is semistable. The skeleton inclusion realizes to an h-cofibration $\left|\mathrm{sk}_{n-1} \amalg_{*} F\right| \longrightarrow\left|\mathrm{sk}_{n} \amalg_{*} F\right|$ whose quotient symmetric spectrum is a wedge, indexed over the nondegenerate $n$-simplices of the nerve of $J$, of $n$-fold suspensions of spectra which occur as values of $F$. So the quotient spectra are semistable, and so by induction the symmetric spectra $\left|\mathrm{sk}_{n} \amalg_{*} F\right|$ are semistable.

As the final result in this section we show that the smash product of two semistable symmetric spectra is again semistable, under a mild cofibrancy condition. We switch to symmetric spectra of simplicial set (as opposed to topological spaces) for the rest of this section, because several results which we will want to quote are in the literature in this context. A symmetric spectrum of simplicial sets $X$ is $S$-cofibrant if for all $n \geq 0$ the natural map $L_{n} X \longrightarrow X_{n}$ is injective. Here $L_{n} X=(X \wedge \bar{S})_{n}$ is the $n$-th latching space in the sense of [3, Definition 5.2.1] (this characterization of $S$ cofibrations does not seem to be in the literature, but the proof is analogous to the proof 
of [3, Proposition 5.2.2] for stable cofibrations). A symmetric spectrum of simplicial sets $X$ is stably cofibrant if for all $n \geq 0$ the natural map $L_{n} X \longrightarrow X_{n}$ is injective and the symmetric group $\Sigma_{n}$ acts freely away from the image. Thus every stably cofibrant symmetric spectrum is $S$-cofibrant, but not vice versa. For example, semifree symmetric spectra $H_{n} L$ associated to a pointed $\Sigma_{n}$-simplicial set $L$ are $S$-cofibrant, but not stably cofibrant, unless the $\Sigma_{n}$-action is free away from the basepoint.

4.9 Proposition Smashing with an $S$-cofibrant symmetric spectrum preserves $\pi_{*}-$ isomorphisms.

Proof We go through a sequence of steps proving that successively larger classes of $S$-cofibrant spectra have the desired property. By Example 3.10 respectively Example 3.13, the homotopy groups of $S^{1} \wedge X$ and of $F_{1} S^{0} \wedge X$ are functors of the homotopy groups of $X$. So smashing with $S^{1}$ and $F_{1} S^{0}$ preserves $\pi_{*}$-isomorphisms. Since $F_{n} S^{m}$ is isomorphic to $\left(F_{1} S^{0}\right)^{\wedge m} \wedge\left(S^{1}\right)^{\wedge n}$, smashing with the cofibrant spectrum $F_{n} S^{m}$ preserves $\pi_{*}$-isomorphisms. Since homotopy groups of a wedge are the direct sum of the homotopy groups, smashing with a wedge of spectra of the form $F_{n} S^{m}$, for varying $n$ and $m$, preserves $\pi_{*}$-isomorphisms.

Every stably cofibrant symmetric spectrum is a retract of one built via the small object argument as $X=\operatorname{colim} X_{n}$, starting with $X_{0}=*$, and such that each $X_{i} \longrightarrow X_{i+1}$ is a stable cofibration with quotient isomorphic to a wedge of symmetric spectra of the form $F_{n} S^{m}$. Inductively, using that cofiber sequences of spectra give rise to long exact sequences of homotopy groups, smashing with each $X_{i}$ preserves $\pi_{*}$-isomorphisms. Thus smashing with the filtered colimit $X$ and thus with an arbitrary stably cofibrant spectrum, preserves $\pi_{*}$-isomorphisms.

Finally, let $X$ be an $S$-cofibrant symmetric spectrum. We choose a level equivalence $X^{\prime} \longrightarrow X$ with stably cofibrant source. If $f: A \longrightarrow B$ is a $\pi_{*}$-isomorphism, then in the commutative square

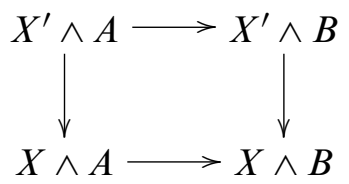

the upper horizontal map is a $\pi_{*}$-isomorphism by the above. Both vertical maps are level equivalences by $\left[3\right.$, Section 5]. Thus the lower map is a $\pi_{*}$-isomorphism, which finishes the proof.

4.10 Theorem Let $X$ and $Y$ be two semistable spectra one of which is $S$-cofibrant. Then the smash product $X \wedge Y$ is semistable. 
Proof Suppose $X$ is $S$-cofibrant and semistable. We first prove the proposition when $Y$ has a special form, namely $Y=\Omega^{n} L^{\prime}\left(\Sigma^{\infty} K\right)$ for a pointed simplicial set $K$, where $L^{\prime}$ is a level fibrant replacement functor. Smashing with an $S$-cofibrant spectrum preserves level equivalences [3, Theorem 5.3.7], so $X \wedge L^{\prime}\left(\Sigma^{\infty} K\right)$ is level equivalent to $X \wedge \Sigma^{\infty} K$, which is isomorphic to the symmetric spectrum $K \wedge X$ and thus semistable by Example 4.7.

The counit of the adjunction between loop and suspension is a $\pi_{*}$-isomorphism $\varepsilon: S^{n} \wedge \Omega^{n} L^{\prime}\left(\Sigma^{\infty} K\right) \longrightarrow L^{\prime}\left(\Sigma^{\infty} K\right)$, so by Proposition 4.9 the map

$$
\text { Id } \wedge \varepsilon: X \wedge S^{n} \wedge \Omega^{n} L^{\prime}\left(\Sigma^{\infty} K\right) \longrightarrow X \wedge L^{\prime}\left(\Sigma^{\infty} K\right)
$$

is a $\pi_{*}$-isomorphism. Since the target is semistable, so is $X \wedge S^{n} \wedge \Omega^{n} L^{\prime}\left(\Sigma^{\infty} K\right)$. A symmetric spectrum is semistable if and only if its suspension is, so we conclude that $X \wedge \Omega^{n} L^{\prime}\left(\Sigma^{\infty} K\right)$ is semistable.

To prove the general case we use Shipley's detection functor [7, Section 3] which associates to every symmetric spectrum $Y$ the semistable symmetric spectrum $D Y$. Here $D Y$ is the homotopy colimit of a functor $\mathcal{D}_{Y}: I \longrightarrow S p^{\Sigma}$ from the category $I$ to the category of symmetric spectra with values $\mathcal{D}_{Y}(\mathbf{n})=\Omega^{n} L^{\prime}\left(\Sigma^{\infty} Y_{n}\right)$. By the above the symmetric spectrum $X \wedge \mathcal{D}_{Y}(\mathbf{n})=X \wedge \Omega^{n} L^{\prime}\left(\Sigma^{\infty} Y_{n}\right)$ is semistable for each $n \geq 0$. Hence the homotopy colimit of the functor $X \wedge \mathcal{D}_{Y}: I \longrightarrow S p^{\Sigma}$, which is isomorphic to $X \wedge D Y$, is semistable by Example 4.8.

By [7, Cor. 3.1.7] the semistable spectrum $Y$ is related by a chain of $\pi_{*}$-isomorphisms to the symmetric spectrum $D Y$. By Proposition 4.9, $X \wedge Y$ is thus related by a chain of $\pi_{*}$-isomorphisms to the symmetric spectrum $X \wedge D Y$, which we just recognized as semistable. Hence $X \wedge Y$ is semistable, which finishes the proof.

\section{Shipley's spectral sequence for true homotopy groups}

By the "true" homotopy groups of a symmetric spectrum $X$ we mean the morphisms in the stable homotopy category from sphere spectra to $X$. In practice, one is usually interested in the true homotopy groups of a symmetric spectrum and not the naively defined and sometimes pathological homotopy groups as in (1.1). However, the naive homotopy groups are more readily computable from an explicit presentation of the symmetric spectrum, so one would like a way to obtain the true homotopy groups from the naive ones.

The true homotopy groups can be calculated as the naive homotopy groups of the target of any stably fibrant replacement, ie, any stable equivalence $X \longrightarrow L X$ whose target 
is an $\Omega$-spectrum. However, stably fibrant replacements are typically obtained by the small object argument, so it is often difficult to get one's hands on their homotopy groups. In the paper [7] Shipley introduces a detection functor $D$ such that for every symmetric spectrum $X$, the naive homotopy groups of $D X$ are naturally isomorphic to the true homotopy groups of $X$. Moreover, the spectrum $D X$ is defined as a homotopy colimit, so it comes with a Bousfield-Kan spectral sequence for calculating its homotopy groups. We now discuss the spectral sequence associated to $D X$ from the perspective of the $M$-action and reinterpret its $E^{2}$-term as Tor groups over the monoid ring of $M$. Then we discuss several examples in which one can completely understand the spectral sequence.

We recall Shipley's construction from [7, Section 3], which is inspired by Bökstedt's use of a homotopy colimit over the category $I$ in the original definition of topological Hochschild homology [1]. First, Shipley associates to a symmetric spectrum $X$ a functor $\mathcal{D}_{X}: I \longrightarrow S p^{\Sigma}$ from the category $I$ of finite sets and injections to the category of symmetric spectra by the rule

$$
\mathcal{D}_{X}(\mathbf{n})=\Omega^{n}\left(F_{0} X_{n}\right) .
$$

(Shipley works simplicially and also needs a level fibrant replacement before taking loops, which is unnecessary in the topological context). Here $F_{0} X_{n}$ is the free symmetric spectrum generated in level 0 by the pointed space $X_{n}$ (so $F_{0} X_{n}$ is another name for the symmetric suspension spectrum $\Sigma^{\infty} X_{n}$ ).

This assignment becomes a functor in $\mathbf{n} \in I$ as follows. The symmetric group $\Sigma_{n}=$ $I(\mathbf{n}, \mathbf{n})$ acts on $\Omega^{n}\left(F_{0} X_{n}\right)=\operatorname{map}\left(S^{n}, F_{0} X_{n}\right)$ by conjugation, using the given action on $X_{n}$ and the coordinate permutations on the source sphere $S^{n}$. The morphism $\mathcal{D}_{X}(\iota): \mathcal{D}_{X}(\mathbf{n}) \longrightarrow \mathcal{D}_{X}(\mathbf{n}+\mathbf{m})$ induced by the inclusion $\iota: \mathbf{n} \longrightarrow \mathbf{n}+\mathbf{m}$ is given in level $k$ as follows. If $f: S^{n} \longrightarrow X_{n} \wedge S^{k}$ is an element of $\mathcal{D}_{X}(\mathbf{n})_{k}=\Omega^{n}\left(X_{n} \wedge S^{k}\right)$, then $\mathcal{D}_{X}(\iota)(f)$ is the composite

$$
S^{n+m} \stackrel{f \wedge \mathrm{Id}}{\longrightarrow} X_{n} \wedge S^{k} \wedge S^{m} \stackrel{\mathrm{Id} \wedge \tau}{\longrightarrow} X_{n} \wedge S^{m} \wedge S^{k} \stackrel{\sigma^{m} \wedge \mathrm{Id}}{\longrightarrow} X_{n+m} \wedge S^{k} .
$$

This uniquely extends to a functor on the category $I$.

Shipley then defines $D X$ as the homotopy colimit of $\mathcal{D}_{X}$ over the category $I$ and proves in [7, Theorem 3.1.6] that $D X$ is related by a natural chain of $\pi_{*}$-isomorphisms to a stably fibrant replacement of $X$. So the naive homotopy groups of $D X$ are isomorphic to the true homotopy groups of $X$.

Since the naive homotopy groups are a homology theory on symmetric spectra, the homotopy colimit $D X$ comes with a Bousfield-Kan spectral sequence [2, Chapter XII, Section 5] converging to the homotopy groups of $D X$ whose $E_{p, q}^{2}$-term equals 
$\operatorname{colim}_{I}^{p} \pi_{q} \mathcal{D}_{X}$, the $p$-th left derived functor of the colimit functor, applied to the $I$-functor $\mathbf{n} \mapsto \pi_{q} \mathcal{D}_{X}(\mathbf{n})$. We have

$$
\pi_{q} \mathcal{D}_{X}(\mathbf{n})=\pi_{q}\left(\Omega^{n} F_{0} X_{n}\right) \cong \pi_{q+n}^{\mathrm{s}} X_{n},
$$

and as $\mathbf{n}$ varies, this yields an isomorphism of $I$-functors $\pi_{q} \mathcal{D}_{X} \cong \underline{\pi}_{q}^{\mathrm{s}} X$. Theorem 5.4 below provides natural isomorphisms of abelian groups

$$
\operatorname{colim}_{I}^{p}\left(\underline{\pi}_{q}^{\mathrm{s}} X\right) \cong \operatorname{Tor}_{p}^{\mathbb{Z}[M]}\left(\mathbb{Z}, \pi_{q} X\right) .
$$

This makes it clear that the derived colimits only depend on the sequential colimit of the functor $\underline{\pi}_{q}^{\mathrm{s}} X$, ie, the homotopy groups of $X$, together with the $M$-action.

Using these isomorphisms, the Bousfield-Kan spectral sequence takes the form of a strongly convergent half-plane spectral sequence

$$
E_{p, q}^{2}=\operatorname{Tor}_{p}^{\mathbb{Z}[M]}\left(\mathbb{Z}, \pi_{q} X\right) \Longrightarrow \pi_{p+q}(D X) .
$$

The spectral sequence is natural in $X$ with $d^{r}$-differential of bidegree $(-r, r-1)$. The edge homomorphism

$$
\mathbb{Z} \otimes_{M} \pi_{q} X=E_{0, q}^{2} \longrightarrow \pi_{q}(D X)
$$

is induced on homotopy groups by the stably fibrant replacement $X \longrightarrow L X$ composed with Shipley's isomorphism [7, Theorem 3.1.6 (1)] between the homotopy groups of $D X$ and those of the stably fibrant replacement $L X$. Here, and below, we use the notation $-\otimes_{M}-$ as short hand for the tensor product over the monoid ring $\mathbb{Z}[M]$.

We will see below that the spectral sequence (5.1) collapses in many cases, for example for semistable symmetric spectra and for free symmetric spectra (see Example 5.5), and it always collapses rationally (see Example 5.7). The spectral sequence typically does not collapse for semifree symmetric spectra; see Example 5.9.

For the identification of derived colimits with Tor groups we need two preparatory Lemmas. I owe the proof of Lemma 5.2 to Neil Strickland.

5.2 Lemma The classifying space $B M$ of the monoid $M$ is contractible.

Proof The classifying space $B M$ is the geometric realization of the nerve of the category $\underline{B} M$ with one object whose monoid of endomorphisms is $M$. Let $t \in M$ be given by $t(i)=2 i$. We define an injective endomorphism $c_{t}: M \longrightarrow M$ as follows. For $f \in M$ and $i \in \omega$ we set

$$
c_{t}(f)(i)=\left\{\begin{array}{cl}
i & \text { if } i \text { is odd, and } \\
2 \cdot f(i / 2) & \text { if } i \text { is even. }
\end{array}\right.
$$


Even though $t$ is not bijective, the endomorphism $c_{t}$ behaves like conjugation by $t$ in the sense that the formula $c_{t}(f) \cdot t=t \cdot f$ holds. Thus $t$ provides a natural transformation from the identity functor of $\underline{B} M$ to $\underline{B}\left(c_{t}\right)$. On the other hand, if $s \in M$ is given by $s(i)=2 i-1$, then $c_{t}(f) \cdot s=s$ for all $f \in M$, so $s$ provides a natural transformation from the constant functor of $\underline{B} M$ with value $1 \in M$ to $\underline{B}\left(c_{t}\right)$. Thus via the homotopies induced by $t$ and $s$, the identity of $B M$ is homotopic to a constant map, so $B M$ is contractible.

5.3 Lemma (i) Let $\mathbb{Z}[M]^{+}$denote the monoid ring of $M$ with its usual left action, but with right action through the monomorphism $(1 \times-): M \longrightarrow M$ given by $(1 \times$ $f)(1)=1$ and $(1 \times f)(i)=f(i-1)+1$ for $i \geq 2$. Then for every $n \geq 0$ the map

$$
\kappa: \mathcal{P}_{1+n} \longrightarrow \mathbb{Z}[M]^{+} \otimes_{M} \mathcal{P}_{n}
$$

which sends the generator $(1, \ldots, n+1)$ to the element $1 \otimes(1, \ldots, n)$ of filtration $n+1$ in $\mathbb{Z}[M]^{+} \otimes_{M} \mathcal{P}_{n}$ is an isomorphism of $M$-modules.

(ii) For every $n \geq 0$ and every abelian group $A$, the groups $\operatorname{Tor}_{p}^{\mathbb{Z}[M]}\left(\mathbb{Z}, \mathcal{P}_{n} \otimes A\right)$ vanish in positive dimensions.

Proof (i) For any $n$-tuple $\left(x_{1}, \ldots, x_{n}\right)$ of pairwise distinct natural numbers we can choose $g \in M$ with $g(i)=x_{i}$ for $1 \leq i \leq n$. Because of

$$
f \otimes\left(x_{1}, \ldots, x_{n}\right)=f \otimes g(1, \ldots, n)=f(1 \times g) \cdot(1 \otimes(1, \ldots, n))
$$

the element $1 \otimes(1, \ldots, n)$ generates $\mathbb{Z}[M]^{+} \otimes_{M} \mathcal{P}_{n}$, so the map $\kappa$ is surjective. The map $\mathbb{Z}[M]^{+} \otimes_{M} \mathcal{P}_{n} \longrightarrow \mathcal{P}_{1+n}$ which sends $f \otimes\left(x_{1}, \ldots, x_{n}\right)$ to $\left(f(1), f\left(x_{1}+\right.\right.$ $\left.1), \ldots, f\left(x_{n}+1\right)\right)$ is right inverse to $\kappa$ since the composite sends the generator $(1, \ldots, n+1)$ to itself. So $\kappa$ is also injective.

(ii) The groups $\operatorname{Tor}_{p}^{\mathbb{Z}[M]}(\mathbb{Z}, A)$ are isomorphic to the singular homology groups with coefficients in $A$ of the classifying space $B M$ of the monoid $M$. This classifying space is contractible by Lemma 5.2, so the groups $\operatorname{Tor}_{p}^{\mathbb{Z}[M]}(\mathbb{Z}, A)$ vanish for $p \geq 1$, which proves the case $n=0$.

For $n \geq 1$, the $M$-modules $\mathcal{P}_{1+n} \otimes A$ and $\mathbb{Z}[M]^{+} \otimes_{M} \mathcal{P}_{n} \otimes A$ are isomorphic by part (i). Since the $M$-bimodule $\mathbb{Z}[M]^{+}$is free as a left and right module separately, the balancing property of Tor groups yields

$$
\begin{aligned}
\operatorname{Tor}_{*}^{\mathbb{Z}[M]}\left(\mathbb{Z}, \mathcal{P}_{1+n} \otimes A\right) & \cong \operatorname{Tor}_{*}^{\mathbb{Z}[M]}\left(\mathbb{Z}, \mathbb{Z}[M]^{+} \otimes_{M} \mathcal{P}_{n} \otimes A\right) \\
& \cong \operatorname{Tor}_{*}^{\mathbb{Z}[M]}\left(\mathbb{Z} \otimes_{M} \mathbb{Z}[M]^{+}, \mathcal{P}_{n} \otimes A\right) \cong \operatorname{Tor}_{*}^{\mathbb{Z}[M]}\left(\mathbb{Z}, \mathcal{P}_{n} \otimes A\right)
\end{aligned}
$$

since $\mathbb{Z} \otimes_{M} \mathbb{Z}[M]^{+}$is again the trivial right $M$-module $\mathbb{Z}$. So induction on $n$ shows that the groups $\operatorname{Tor}_{p}^{\mathbb{Z}[M]}\left(\mathbb{Z}, \mathcal{P}_{n} \otimes A\right)$ vanish in positive dimensions. 
5.4 Theorem For every $I$-functor $F$ there are natural isomorphisms of abelian groups

$$
\operatorname{colim}_{I}^{p} F \cong \operatorname{Tor}_{p}^{\mathbb{Z}[M]}\left(\mathbb{Z}, \operatorname{colim}_{\mathbb{N}} F\right)
$$

for all $p \geq 0$.

Proof We show that the collection of functors

$$
\left\{F \mapsto \operatorname{Tor}_{p}^{\mathbb{Z}[M]}\left(\mathbb{Z}, \operatorname{colim}_{\mathbb{N}} F\right)\right\}_{p \geq 0}
$$

has all the properties that characterize the left derived functors of the colimit. Clearly

$$
\operatorname{colim}_{I} F \cong \mathbb{Z} \otimes_{M}\left(\operatorname{colim}_{\mathbb{N}} F\right)
$$

so the functors agree for $p=0$. Taking colimit over the filtered category $\mathbb{N}$ is exact, so the Tor functors of the colimit take short exact sequences of $I$-functors to long exact sequences of abelian groups. The least obvious part is that the Tor groups $\operatorname{Tor}_{p}^{\mathbb{Z}[M]}\left(\mathbb{Z}, \operatorname{colim}_{\mathbb{N}} F\right)$ vanish for all projective functors $F$ and all $p \geq 1$. The $I-$ functors $\mathbb{Z}[I(\mathbf{n},-)]$ arising as the linearizations of the representable functors form a set of projective generators for the category of $I$-functors, so it suffices to show the vanishing of higher Tor groups for these. But the colimit over $\mathbb{N}$ of the $I$-functor $\mathbb{Z}[I(\mathbf{n},-)]$ is precisely the $M$-module $\mathcal{P}_{n}$; so Lemma 5.3 (ii) provides the vanishing result and finishes the proof.

5.5 Example (Semistable and free symmetric spectra) When $X$ is a semistable symmetric spectrum or a free symmetric spectrum, then the higher Tor groups for the homotopy of $X$ vanish by Lemma 5.3 (ii). Thus in the spectral sequence (5.1) we have $E_{p, q}^{2}=0$ for $p \neq 0$, and so the edge homomorphism

$$
\mathbb{Z} \otimes_{M}\left(\pi_{*} X\right) \longrightarrow \pi_{*}(D X)
$$

is an isomorphism.

5.6 Example (Eilenberg-Mac Lane spectra) In Example 3.3 we associate an Eilenberg-Mac Lane spectrum $H W$ to every tame $M$-module $W$. The homotopy groups of $H W$ are concentrated in dimension 0 , where we get the module $W$ back. So the spectral sequence (5.1) for $H W$ collapses onto the axis $q=0$ to isomorphisms

$$
\pi_{p}(D(H W)) \cong \operatorname{Tor}_{p}^{\mathbb{Z}[M]}(\mathbb{Z}, W) .
$$

In particular, the true homotopy groups of $H W$ need not be concentrated in dimension 0 .

Here is an example which shows that for nontrivial $W$ the Eilenberg-Mac Lane spectrum $H W$ can be stably contractible: we let $W$ be the kernel of a surjection 
$\mathcal{P}_{n} \longrightarrow \mathbb{Z}$. Lemma 5.3 (ii) and the long exact sequence of Tor groups show that the groups $\operatorname{Tor}_{p}^{\mathbb{Z}[M]}(\mathbb{Z}, W)$ vanish for all $p \geq 0$. Thus the homotopy groups of $D(H W)$ are trivial, ie, $H W$ is stably contractible.

5.7 Example (Rational collapse) We claim that for every tame $M$-module $W$ and all $p \geq 1$, we have $\operatorname{Tor}_{p}^{\mathbb{Z}[M]}(\mathbb{Q}, W)=0$. So the spectral sequence (5.1) always collapses rationally and the edge homomorphism is a rational isomorphism

$$
\mathbb{Q} \otimes_{M}\left(\pi_{*} X\right) \longrightarrow \mathbb{Q} \otimes \pi_{*}(D X) .
$$

The rational vanishing of higher Tor groups is special for tame $M$-modules.

To prove the claim we consider a monomorphism $i: V \longrightarrow W$ of tame $M$-modules and show that the kernel of the map $\mathbb{Z} \otimes_{M} i: \mathbb{Z} \otimes_{M} V \longrightarrow \mathbb{Z} \otimes_{M} W$ is a torsion group. The inclusions $W^{(n)} \longrightarrow W$ induce an isomorphism

$$
\operatorname{colim}_{n} \mathbb{Z} \otimes_{\Sigma_{n}} W^{(n)} \stackrel{\cong}{\longrightarrow} \mathbb{Z} \otimes_{M} W .
$$

For every $n \geq 0$, the kernel of $\mathbb{Z} \otimes \Sigma_{n} i^{(n)}: \mathbb{Z} \otimes \Sigma_{n} V^{(n)} \longrightarrow \mathbb{Z} \otimes \Sigma_{n} W^{(n)}$ is annihilated by the order of the group $\Sigma_{n}$. Since the kernel of $\mathbb{Z} \otimes_{M} i$ is the colimit of the kernels of the maps $\mathbb{Z} \otimes_{\Sigma_{n}} i^{(n)}$, it is torsion. Thus the functor $\mathbb{Q} \otimes_{M}-$ is exact on short exact sequences of tame $M$-modules and the higher Tor groups vanish as claimed.

The Tor groups discussed in the next lemma arise in the spectral sequence (5.1) for semifree symmetric spectra.

5.8 Lemma For every $\Sigma_{n}$-module $B$ we have a natural isomorphism

$$
\operatorname{Tor}_{*}^{\mathbb{Z}[M]}\left(\mathbb{Z}, \mathcal{P}_{n} \otimes_{\Sigma_{n}} B\right) \cong H_{*}\left(\Sigma_{n} ; B\right)
$$

Proof Since $\mathcal{P}_{n}$ is free as a right $\Sigma_{n}$-module, the functor $\mathcal{P}_{n} \otimes \Sigma_{n}-$ is exact. The functor takes the free $\Sigma_{n}$-module of rank 1 to $\mathcal{P}_{n}$, so by Lemma 5.3 (ii) it takes projective $\Sigma_{n}$-modules to tame $M$-modules which are acyclic for the functor $\mathbb{Z} \otimes_{M}-$.

Thus if $P_{\bullet} \longrightarrow B$ is a projective resolution of $B$ by $\Sigma_{n}$-modules, then $\mathcal{P}_{n} \otimes_{\Sigma_{n}} P_{\bullet}$ is a resolution of $\mathcal{P}_{n} \otimes \Sigma_{n} B$ which can be used to calculate the desired Tor groups. Thus we have isomorphisms

$$
\operatorname{Tor}_{*}^{\mathbb{Z}[M]}\left(\mathbb{Z}, \mathcal{P}_{n} \otimes_{\Sigma_{n}} B\right)=H_{*}\left(\mathbb{Z} \otimes_{M} \mathcal{P}_{n} \otimes_{\Sigma_{n}} P_{\bullet}\right) \cong H_{*}\left(\mathbb{Z} \otimes_{\Sigma_{n}} P_{\bullet}\right)=H_{*}\left(\Sigma_{n} ; B\right) .
$$

5.9 Example (Semifree symmetric spectra) For semifree symmetric spectra (see Example 3.6) the spectral sequence (5.1) typically does not degenerate. As an example 
we consider the semifree symmetric spectrum $H_{2} S^{2}$, where $S^{2}$ is a $\Sigma_{2}$-space by coordinate permutations.

We first identify the stable equivalence type of $\mathrm{H}_{2} \mathrm{~S}^{2}$. The spectrum $\mathrm{H}_{2} \mathrm{~S}^{2}$ is isomorphic to the quotient spectrum of $\Sigma_{2}$ permuting the smash factors of $\left(F_{1} S^{1}\right)^{\wedge 2}$. Since the $\Sigma_{2}$-action on $\left(F_{1} S^{1}\right)^{\wedge 2}$ is free, the map

$$
\left(F_{1} S^{1}\right)^{\wedge 2} \wedge_{\Sigma_{2}} E \Sigma_{2}^{+} \longrightarrow\left(F_{1} S^{1}\right)^{\wedge 2} / \Sigma_{2}=H_{2} S^{2}
$$

which collapses $E \Sigma_{2}$ to a point is a level equivalence. On the other hand, the stable equivalence $\lambda^{\wedge 2}$ : $\left(F_{1} S^{1}\right)^{\wedge 2} \longrightarrow S$ is $\Sigma_{2}$-equivariant, so it induces a stable equivalence

$$
\left(F_{1} S^{1}\right)^{\wedge 2} \wedge \Sigma_{2} E \Sigma_{2}^{+} \longrightarrow S \wedge \Sigma_{2} E \Sigma_{2}^{+}=\Sigma^{\infty} B \Sigma_{2}^{+}
$$

on homotopy orbit spectra. Altogether we conclude that $\mathrm{H}_{2} \mathrm{~S}^{2}$ is stably equivalent to $\Sigma^{\infty} B \Sigma_{2}^{+}$.

The spectral sequence (5.1) for $\mathrm{H}_{2} \mathrm{~S}^{2}$ has as $E^{2}$-term the Tor groups of $\pi_{*}\left(\mathrm{H}_{2} \mathrm{~S}^{2}\right)$. According to (3.7) these homotopy groups are isomorphic to $\mathcal{P}_{2} \otimes_{\Sigma_{2}}\left(\pi_{*+2}^{\mathrm{s}} S^{2}\right)(\mathrm{sgn})$. The sign representation cancels the sign action induced by the coordinate flip of $S^{2}$, so we have an isomorphism of $M$-modules $\pi_{q}\left(H_{2} S^{2}\right) \cong \mathcal{P}_{2} \otimes_{\Sigma_{2}} \pi_{q}^{\mathrm{s}} S^{0}$, this time with trivial action on the stable homotopy groups of spheres. Using Lemma 5.8, the spectral sequence (5.1) for $\mathrm{H}_{2} \mathrm{~S}^{2}$ takes the form

$$
E_{p, q}^{2} \cong H_{p}\left(\Sigma_{2} ; \pi_{q}^{\mathrm{s}} S^{0}\right) \Longrightarrow \pi_{p+q}^{\mathrm{s}}\left(B \Sigma_{2}^{+}\right) .
$$

This spectral sequence has nontrivial differentials and it seems likely that it coincides with the Atiyah-Hirzebruch spectral sequence for the stable homotopy of the space $B \Sigma_{2}^{+}$.

\section{References}

[1] M Bökstedt, Topological Hochschild homology, preprint, Bielefeld (1985)

[2] A K Bousfield, D M Kan, Homotopy limits, completions and localizations, Lecture Notes in Math. 304, Springer, Berlin (1972) MR0365573

[3] M Hovey, B Shipley, J Smith, Symmetric spectra, J. Amer. Math. Soc. 13 (2000) 149-208 MR1695653

[4] M Lydakis, Smash products and $\Gamma$-spaces, Math. Proc. Cambridge Philos. Soc. 126 (1999) 311-328 MR1670245

[5] M A Mandell, J P May, S Schwede, B Shipley, Model categories of diagram spectra, Proc. London Math. Soc. (3) 82 (2001) 441-512 MR1806878 
[6] G Segal, Categories and cohomology theories, Topology 13 (1974) 293-312 MR0353298

[7] B Shipley, Symmetric spectra and topological Hochschild homology, K-Theory 19 (2000) 155-183 MR1740756

Mathematisches Institut, Universität Bonn

Beringstraße 3, 53115 Bonn, Germany

schwede@math.uni-bonn.de

Proposed: Bill Dwyer

Received: 30 September 2006

Seconded: Peter Teichner, Ralph Cohen

Accepted: 5 April 2008

Geometry 8 Topology, Volume 12 (2008) 